\title{
DISCONTINUOUS GALERKIN METHODS FOR THE VLASOV-MAXWELL EQUATIONS
}

\author{
YINGDA CHENG*, IRENE M. GAMBA ${ }^{\dagger}$, FENGYAN LI ${ }^{\ddagger}$, AND PHILIP J. MORRISON ${ }^{\S}$
}

\begin{abstract}
Discontinuous Galerkin methods are developed for solving the Vlasov-Maxwell system, methods that are designed to be systematically as accurate as one wants with provable conservation of mass and possibly total energy. Such properties in general are hard to achieve within other numerical method frameworks for simulating the Vlasov-Maxwell system. The proposed scheme employs discontinuous Galerkin discretizations for both the Vlasov and the Maxwell equations, resulting in a consistent description of the distribution function and electromagnetic fields. It is proven, up to some boundary effects, that charge is conserved and the total energy can be preserved with suitable choices of the numerical flux for the Maxwell equations and the underlying approximation spaces. Error estimates are established for several flux choices. The scheme is tested on the streaming Weibel instability: the order of accuracy and conservation properties of the proposed method are verified.
\end{abstract} bility

Key words. Vlasov-Maxwell system, discontinuous Galerkin methods, energy conservation, error estimates, Weibel insta-

AMS subject classifications. 65M60, 74S05

1. Introduction. In this paper, we consider the Vlasov-Maxwell (VM) system, the most important equation for the modeling of collisionless magnetized plasmas. In particular, we study the evolution of a single species of nonrelativistic electrons under the self-consistent electromagnetic field while the ions are treated as uniform fixed background. Under the scaling of the characteristic time by the inverse of the plasma frequency $\omega_{p}^{-1}$, length by the Debye length $\lambda_{D}$, and electric and magnetic fields by $-m c \omega_{p} / e$ (with $m$ the electron mass, $c$ the speed of light, and $e$ the electron charge), the dimensionless form of the VM system is

$$
\begin{gathered}
\partial_{t} f+\xi \cdot \nabla_{\mathbf{x}} f+(\mathbf{E}+\xi \times \mathbf{B}) \cdot \nabla_{\xi} f=0, \\
\frac{\partial \mathbf{E}}{\partial t}=\nabla_{\mathbf{x}} \times \mathbf{B}-\mathbf{J}, \quad \frac{\partial \mathbf{B}}{\partial t}=-\nabla_{\mathbf{x}} \times \mathbf{E}, \\
\nabla_{\mathbf{x}} \cdot \mathbf{E}=\rho-\rho_{i}, \quad \nabla_{\mathbf{x}} \cdot \mathbf{B}=0,
\end{gathered}
$$

with

$$
\rho(\mathbf{x}, t)=\int_{\Omega_{\xi}} f(\mathbf{x}, \xi, t) d \xi, \quad \mathbf{J}(\mathbf{x}, t)=\int_{\Omega_{\xi}} f(\mathbf{x}, \xi, t) \xi d \xi,
$$

where the equations are defined on $\Omega=\Omega_{x} \times \Omega_{\xi}, \mathbf{x} \in \Omega_{x}$ denotes position in physical space, and $\xi \in \Omega_{\xi}$ in velocity space. Here $f(\mathbf{x}, \xi, t) \geq 0$ is the distribution function of electrons at position $\mathbf{x}$ with velocity $\xi$ at time $t, \mathbf{E}(\mathbf{x}, t)$ is the electric field, $\mathbf{B}(\mathbf{x}, t)$ is the magnetic field, $\rho(\mathbf{x}, t)$ is the electron charge density, and $\mathbf{J}(\mathbf{x}, t)$ is the current density. The charge density of background ions is denoted by $\rho_{i}$, which is chosen to satisfy total charge neutrality, $\int_{\Omega_{x}}\left(\rho(\mathbf{x}, t)-\rho_{i}\right) d \mathbf{x}=0$. Periodic boundary conditions in $\mathbf{x}$-space are assumed and the initial conditions are denoted by $f_{0}=f(\mathbf{x}, \xi, 0), \mathbf{E}_{0}=\mathbf{E}(\mathbf{x}, 0)$ and $\mathbf{B}_{0}=\mathbf{B}(\mathbf{x}, 0)$. We also assume that the initial distribution function $f_{0}(\mathbf{x}, \xi) \in H^{m}(\Omega) \cap L_{2}^{1}\left(\Omega_{\xi}\right)$, i.e., is in a Sobolev space of order $m$ and is integrable with finite energy in $\xi$-space, where $L_{m}^{p}\left(\Omega_{\xi}\right) \equiv\left\{\psi: \int_{\Omega_{\xi}}|\psi|^{p}\left(1+|\xi|^{2}\right)^{m / 2} d \xi<\infty\right\}$. The initial fields $\mathbf{E}_{0}(\mathbf{x})$ and $\mathbf{B}_{0}(\mathbf{x})$ are also assumed to be in $H^{m}\left(\Omega_{x}\right)$.

The VM system has wide importance in plasma physics for describing space and laboratory plasmas, with application to fusion devices, high-power microwave generators, and large scale particle accelerators. The computation of the initial boundary value problem associated to the VM system is quite challenging, due to the high-dimensionality (6D+time) of the Vlasov equation, multiple temporal and spatial scales associated with various physical phenomena, nonlinearity, and the conservation of physical quantities due to the Hamiltonian structure [43, 44] of the system. Particle-in-cell (PIC) methods [5, 35] have long been very popular numerical tools, in which the particles are advanced in a Lagrangian framework, while the field

\footnotetext{
*Department of Mathematics, Michigan State University, East Lansing, MI 48824 U.S.A. ycheng@math.msu.edu

${ }^{\dagger}$ Department of Mathematics and ICES, University of Texas at Austin, Austin, TX 78712 U.S.A. gamba@math.utexas.edu

${ }^{\ddagger}$ Department of Mathematical Sciences, Rensselaer Polytechnic Institute, Troy, NY 12180, U.S.A. lif@rpi.edu

$\S$ Department of Physics and Institute for Fusion Studies, University of Texas at Austin, Austin, TX 78712 U.S.A. morrison@physics. utexas.edu
} 
equations are solved on a mesh. This remains an active area of research [22. In recent years, there has been growing interest in computing the Vlasov equation in a deterministi c framework. In the context of the Vlasov-Poisson system, semi-Lagrangian methods [11, 55], finite volume (flux balance) methods [6, 23, 24, Fourier-Fourier spectral methods [39, 40], and continuous finite element methods [58, 59] have been proposed, among many others. In the context of VM simulations, Califano et al. have used a semi-Lagrangian approach to compute the streaming Weibel (SW) instability [9], current filamentation instability [4], magnetic vortices [8], magnetic reconnection [7]. Also, various methods have been proposed for the relativistic VM system [54, 4, 56, 36.

In this paper, we propose the use of discontinuous Galerkin (DG) methods for solving the VM system. What motivates us to choose DG methods, besides their many widely recognized desirable properties, is that they can be designed systematically to be as accurate as one wants, meanwhile with provable conservation of mass and possibly also the total energy. This is in general hard to achieve within other numerical method frameworks for simulating the VM system. The proposed scheme employs DG discretizations for both the Vlasov and the Maxwell equations, resulting in a consistent description of the distribution function and electromagnetic fields. We will show that up to some boundary effects, depending on the size of the computational domain, the total charge (mass) is conserved and the total energy can be preserved with a suitable choice of the numerical flux for the Maxwell equations and underlying approximation spaces. Error estimates are further established for several flux choices. The DG scheme can be implemented on both structured and unstructured meshes with provable accuracy and stability for many linear and nonlinear problems, it is advantageous in long time wave-like simulations because it has low dispersive and dissipative errors [1, and it is very suitable for adaptive and parallel implementations. The original DG method was introduced by Reed and Hill [51] for a neutron transport equation. Lesaint and Raviart [41] performed the first error estimates for the original DG method, while Cockburn and Shu in a series of papers [18, 17, 16. 15, 19] developed the Runge-Kutta DG (RKDG) methods for hyperbolic equations. RKDG methods have been used to simulate the Vlasov-Poisson system in plasmas [34, 33, 13, and for a gravitational infinite homogeneous stellar system [12. Some theoretical aspects about stability, accuracy and conservation of these methods in their semi-discrete form are discussed in [33, 3, 2]. Recently, semi-Lagrangian DG methods [52, 50] were proposed for the Vlasov-Poisson system. In [37, 38], DG discretizations for Maxwell's equations were coupled with PIC methods to solve the VM system. In a recent work [?], error estimates of fully discrete RKDG methods are studied for the VM system.

The rest of the paper is organized as follows: in Section 2, we describe the numerical algorithm. In Section 3, conservation and the stability are established for the method. In Section 4 , we provide the error estimates of the scheme. Section 5 is devoted to discussion of simulation results. We conclude with a few remarks in Section 6 .

2. Numerical Methods. In this section, we will introduce the DG algorithm for the VM system. We consider an infinite, homogeneous plasma, where all boundary conditions in $\mathbf{x}$ are periodic, and $f(\mathbf{x}, \xi, t)$ is assumed to be compactly supported in $\xi$. This assumption is consistent with the fact that the solution of the VM system is expected to decay at infinity in $\xi$-space, preserving integrability and its kinetic energy.

Without loss of generality, we assume $\Omega_{x}=\left(-L_{x}, L_{x}\right]^{d_{x}}$ and $\Omega_{\xi}=\left[-L_{\xi}, L_{\xi}\right]^{d_{\xi}}$, where the velocity space domain $\Omega_{\xi}$ is chosen large enough so that $f=0$ at and near the phase space boundaries. We take $d_{x}=d_{\xi}=3$ in the following sections, although the method and its analysis can be extended directly to the cases when $d_{x}$ and $d_{\xi}$ take any values from $\{1,2,3\}$.

In our analysis, the assumption that $f(\mathbf{x}, \xi, t)$ remain compactly support in $\xi$, given that it is initially so, is an open question in the general setting. The answer to this question is important for proving the existence of a globally defined classical solution, and its failure could indicate the formation of shock-like solutions of the VM system. Whether or not the three-dimensional VM system is globally well-posed as a Cauchy problem is a major open problem. The limited results of global existence without uniqueness of weak solutions and well-posedness and regularity of solutions assuming either some symmetry or near neutrality constitute the present extent of knowledge [29, 30, 25, 21, 26, 28, 27].

2.1. Notations. Throughout the paper, standard notations will be used for the Sobolev spaces. Given a bounded domain $D \in \mathbb{R}^{\star}$ (with $\star=d_{x}, d_{\xi}$, or $d_{x}+d_{\xi}$ ) and any nonnegative integer $m, H^{m}(D)$ denotes the $L^{2}$-Sobolev space of order $m$ with the standard Sobolev norm $\|\cdot\|_{m, D}$, and $W^{m, \infty}(D)$ denotes the $L^{\infty}$ Sobolev space of order $m$ with the standard Sobolev norm $\|\cdot\|_{m, \infty, D}$ and the semi-norm $|\cdot|_{m, \infty, D}$. When 
$m=0$, we also use $H^{0}(D)=L^{2}(D)$ and $W^{0, \infty}(D)=L^{\infty}(D)$.

Let $\mathcal{T}_{h}^{x}=\left\{K_{x}\right\}$ and $\mathcal{T}_{h}^{\xi}=\left\{K_{\xi}\right\}$ be partitions of $\Omega_{x}$ and $\Omega_{\xi}$, respectively, with $K_{x}$ and $K_{\xi}$ being (rotated) Cartesian elements or simplices; then $\mathcal{T}_{h}=\left\{K: K=K_{x} \times K_{\xi}, \forall K_{x} \in \mathcal{T}_{h}^{x}, \forall K_{\xi} \in \mathcal{T}_{h}^{\xi}\right\}$ defines a partition of $\Omega$. Let $\mathcal{E}_{x}$ be the set of the edges of $\mathcal{T}_{h}^{x}$ and $\mathcal{E}_{\xi}$ be the set of the edges of $\mathcal{T}_{h}^{\xi}$; then the edges of $\mathcal{T}_{h}$ will be $\mathcal{E}=\left\{K_{x} \times e_{\xi}: \forall K_{x} \in \mathcal{T}_{h}^{x}, \forall e_{\xi} \in \mathcal{E}_{\xi}\right\} \cup\left\{e_{x} \times K_{\xi}: \forall e_{x} \in \mathcal{E}_{x}, \forall K_{\xi} \in \mathcal{T}_{h}^{\xi}\right\}$. Here we take into account the periodic boundary condition in the x-direction when defining $\mathcal{E}_{x}$ and $\mathcal{E}$. Furthermore, $\mathcal{E}_{\xi}=\mathcal{E}_{\xi}^{i} \cup \mathcal{E}_{\xi}^{b}$ with $\mathcal{E}_{\xi}^{i}$ and $\mathcal{E}_{\xi}^{b}$ being the set of interior and boundary edges of $\mathcal{T}_{h}^{\xi}$, respectively. In addition, we denote the mesh size of $\mathcal{T}_{h}$ as $h=\max \left(h_{x}, h_{\xi}\right)=\max _{K \in \mathcal{T}_{h}} h_{K}$, where $h_{x}=\max _{K_{x} \in \mathcal{T}_{h}^{x}} h_{K_{x}}$ with $h_{K_{x}}=\operatorname{diam}\left(K_{x}\right)$, $h_{\xi}=\max _{K_{\xi} \in \mathcal{T}_{h}^{\xi}} h_{K_{\xi}}$ with $h_{K_{\xi}}=\operatorname{diam}\left(K_{\xi}\right)$, and $h_{K}=\max \left(h_{K_{x}}, h_{K_{\xi}}\right)$ for $K=K_{x} \times K_{\xi}$. When the mesh is refined, we assume both $\frac{h_{x}}{h_{\xi, \min }}$ and $\frac{h_{\xi}}{h_{x, \min }}$ are uniformly bounded from above by a positive constant $\sigma_{0}$. Here $h_{x, \min }=\min _{K_{x} \in \mathcal{T}_{h}^{x}} h_{K_{x}}$ and $h_{\xi, \min }=\min _{K_{\xi} \in \mathcal{T}_{h}^{\xi}} h_{K_{\xi}}$. It is further assumed that $\left\{\mathcal{T}_{h}^{\star}\right\}_{h}$ is shape-regular with $\star=x$ or $\xi$. That is, if $\rho_{K_{\star}}$ denotes the diameter of the largest sphere included in $K_{\star}$, there is

$$
\frac{h_{K_{\star}}}{\rho_{K_{\star}}} \leq \sigma_{\star}, \quad \forall K_{\star} \in \mathcal{T}_{h}^{\star}
$$

for a positive constant $\sigma_{\star}$ independent of $h_{\star}$.

Next we define the discrete spaces

$$
\begin{aligned}
& \mathcal{G}_{h}^{k}=\left\{g \in L^{2}(\Omega):\left.g\right|_{K=K_{x} \times K_{\xi}} \in P^{k}\left(K_{x} \times K_{\xi}\right), \forall K_{x} \in \mathcal{T}_{h}^{x}, \forall K_{\xi} \in \mathcal{T}_{h}^{\xi}\right\}, \\
& =\left\{g \in L^{2}(\Omega):\left.g\right|_{K} \in P^{k}(K), \forall K \in \mathcal{T}_{h}\right\}, \\
& \mathcal{U}_{h}^{r}=\left\{\mathbf{U} \in\left[L^{2}\left(\Omega_{x}\right)\right]^{d_{x}}:\left.\mathbf{U}\right|_{K_{x}} \in\left[P^{r}\left(K_{x}\right)\right]^{d_{x}}, \forall K_{x} \in \mathcal{T}_{h}^{x}\right\},
\end{aligned}
$$

where $P^{r}(D)$ denotes the set of polynomials of total degree at most $r$ on $D$, and $k$ and $r$ are nonnegative integers. Note the space $\mathcal{G}_{h}^{k}$, which we use to approximate $f$, is called P-type, and it can be replaced by the tensor product of P-type spaces in $\mathbf{x}$ and $\xi$,

$$
\left\{g \in L^{2}(\Omega):\left.g\right|_{K=K_{x} \times K_{\xi}} \in P^{k}\left(K_{x}\right) \times P^{k}\left(K_{\xi}\right), \forall K_{x} \in \mathcal{T}_{h}^{x}, \forall K_{\xi} \in \mathcal{T}_{h}^{\xi}\right\},
$$

or by the tensor product space in each variable, which is called Q-type

$$
\left\{g \in L^{2}(\Omega):\left.g\right|_{K=K_{x} \times K_{\xi}} \in Q^{k}\left(K_{x}\right) \times Q^{k}\left(K_{\xi}\right), \forall K_{x} \in \mathcal{T}_{h}^{x}, \forall K_{\xi} \in \mathcal{T}_{h}^{\xi}\right\} .
$$

Here $Q^{r}(D)$ denotes the set of polynomials of degree at most $r$ in each variable on $D$. The numerical methods formulated in this paper, as well as the conservation, stability, and error estimates, hold when any of the spaces above is used to approximate $f$. In our simulations of Section 5, we use the P-type of (2.1a) as it is the smallest and therefore renders the most cost efficient algorithm. In fact, the ratios of these three spaces defined in 2.1a), 2.2 and 2.3 are $\sum_{n=0}^{k}\left(\begin{array}{c}n+2 d-1 \\ 2 d-1\end{array}\right):\left(\sum_{n=0}^{k}\left(\begin{array}{c}n+d-1 \\ d-1\end{array}\right)\right)^{2}:(k+1)^{2 d}$ with $d_{x}=d_{\xi}=d$.

For piecewise functions defined with respect to $\mathcal{T}_{h}^{x}$ or $\mathcal{T}_{h}^{\xi}$, we further introduce the jumps and averages as follows. For any edge $e=\left\{K_{x}^{+} \cap K_{x}^{-}\right\} \in \mathcal{E}_{x}$, with $\mathbf{n}_{x}^{ \pm}$as the outward unit normal to $\partial K_{x}^{ \pm}, g^{ \pm}=\left.g\right|_{K_{x}^{ \pm}}$, and $\mathbf{U}^{ \pm}=\left.\mathbf{U}\right|_{K_{x}^{ \pm}}$, the jumps across $e$ are defined as

$$
[g]_{x}=g^{+} \mathbf{n}_{x}^{+}+g^{-} \mathbf{n}_{x}^{-}, \quad[\mathbf{U}]_{x}=\mathbf{U}^{+} \cdot \mathbf{n}_{x}^{+}+\mathbf{U}^{-} \cdot \mathbf{n}_{x}^{-}, \quad[\mathbf{U}]_{\tau}=\mathbf{U}^{+} \times \mathbf{n}_{x}^{+}+\mathbf{U}^{-} \times \mathbf{n}_{x}^{-}
$$

and the averages are

$$
\{g\}_{x}=\frac{1}{2}\left(g^{+}+g^{-}\right), \quad\{\mathbf{U}\}_{x}=\frac{1}{2}\left(\mathbf{U}^{+}+\mathbf{U}^{-}\right) .
$$

By replacing the subscript $x$ with $\xi$, one can define $[g]_{\xi},[\mathbf{U}]_{\xi},\{g\}_{\xi}$, and $\{\mathbf{U}\}_{\xi}$ for an interior edge of $\mathcal{T}_{h}^{\xi}$ in $\mathcal{E}_{\xi}^{i}$. For a boundary edge $e \in \mathcal{E}_{\xi}^{b}$ with $\mathbf{n}_{\xi}$ being the outward unit normal, we use

$$
[g]_{\xi}=g \mathbf{n}_{\xi}, \quad\{g\}_{\xi}=\frac{1}{2} g, \quad\{\mathbf{U}\}_{\xi}=\frac{1}{2} \mathbf{U} .
$$


This is consistent with the fact that the exact solution $f$ is compactly supported in $\xi$.

For convenience, we introduce some shorthand notations, $\int_{\Omega_{\star}}=\int_{\mathcal{T}_{h}^{\star}}=\sum_{K_{\star} \in \mathcal{T}_{h}^{\star}} \int_{K_{\star}}, \quad \int_{\Omega}=\int_{\mathcal{T}_{h}}=$ $\sum_{K \in \mathcal{T}_{h}} \int_{K}, \quad \int_{\mathcal{E}_{\star}}=\sum_{e \in \mathcal{E}_{\star}} \int_{e}$, where again $\star$ is $x$ or $\xi$. In addition, $\|g\|_{0, \mathcal{E}}=\left(\|g\|_{0, \mathcal{E}_{x} \times \mathcal{T}_{h}^{\xi}}^{2}+\|g\|_{0, \mathcal{T}_{h}^{x} \times \mathcal{E}_{\xi}}^{2}\right)^{1 / 2}$ with $\|g\|_{0, \mathcal{E}_{x} \times \mathcal{T}_{h}^{\xi}}=\left(\int_{\mathcal{E}_{x}} \int_{\mathcal{T}_{h}^{\xi}} g^{2} d \xi d s_{x}\right)^{1 / 2}, \quad\|g\|_{0, \mathcal{T}_{h}^{x} \times \mathcal{E}_{\xi}}=\left(\int_{\mathcal{T}_{h}^{x}} \int_{\mathcal{E}_{\xi}} g^{2} d s_{\xi} d \mathbf{x}\right)^{1 / 2}$, and $\|g\|_{0, \mathcal{E}_{x}}=\left(\int_{\mathcal{E}_{x}} g^{2} d s_{x}\right)^{1 / 2}$. There are several equalities that will be used later, which can be easily verified using the definitions of averages and jumps.

$$
\begin{gathered}
\frac{1}{2}\left[g^{2}\right]_{\star}=\{g\}_{\star}[g]_{\star}, \text { with } \star=x \text { or } \xi, \\
{[\mathbf{U} \times \mathbf{V}]_{x}+\{\mathbf{V}\}_{x} \cdot[\mathbf{U}]_{\tau}-\{\mathbf{U}\}_{x} \cdot[\mathbf{V}]_{\tau}=0,} \\
{[\mathbf{U} \times \mathbf{V}]_{x}+\mathbf{V}^{+} \cdot[\mathbf{U}]_{\tau}-\mathbf{U}^{-} \cdot[\mathbf{V}]_{\tau}=0, \quad[\mathbf{U} \times \mathbf{V}]_{x}+\mathbf{V}^{-} \cdot[\mathbf{U}]_{\tau}-\mathbf{U}^{+} \cdot[\mathbf{V}]_{\tau}=0 .}
\end{gathered}
$$

We end this subsection by summarizing some standard approximation properties of the above discrete spaces, as well as some inverse inequalities 14. For any nonnegative integer $m$, let $\Pi^{m}$ be the $L^{2}$ projection onto $\mathcal{G}_{h}^{m}$, and $\boldsymbol{\Pi}_{x}^{m}$ be the $L^{2}$ projection onto $\mathcal{U}_{h}^{m}$, then

LEMma 2.1 (Approximation properties). There exists a constant $C>0$, such that for any $g \in H^{m+1}(\Omega)$ and $\mathbf{U} \in\left[H^{m+1}\left(\Omega_{x}\right)\right]^{d_{x}}$, the following hold:

$$
\begin{aligned}
\left\|g-\Pi^{m} g\right\|_{0, K}+h_{K}^{1 / 2}\left\|g-\Pi^{m} g\right\|_{0, \partial K} & \leq C h_{K}^{m+1}\|g\|_{m+1, K}, \quad \forall K \in \mathcal{T}_{h}, \\
\left\|\mathbf{U}-\boldsymbol{\Pi}_{x}^{m} \mathbf{U}\right\|_{0, K_{x}}+h_{K_{x}}^{1 / 2}\left\|\mathbf{U}-\boldsymbol{\Pi}_{x}^{m} \mathbf{U}\right\|_{0, \partial K_{x}} & \leq C h_{K_{x}}^{m+1}\|\mathbf{U}\|_{m+1, K_{x}}, \quad \forall K_{x} \in \mathcal{T}_{h}^{x}, \\
\left\|\mathbf{U}-\boldsymbol{\Pi}_{x}^{m} \mathbf{U}\right\|_{0, \infty, K_{x}} & \leq C h_{K_{x}}^{m+1}\|\mathbf{U}\|_{m+1, \infty, K_{x}}, \quad \forall K_{x} \in \mathcal{T}_{h}^{x},
\end{aligned}
$$

where the constant $C$ is independent of the mesh sizes $h_{K}$ and $h_{K_{x}}$, but depends on $m$ and the shape regularity parameters $\sigma_{x}$ and $\sigma_{\xi}$ of the mesh.

Lemma 2.2 (Inverse inequality). There exists a constant $C>0$, such that for any $g \in P^{m}(K)$ or $P^{m}\left(K_{x}\right) \times P^{m}\left(K_{\xi}\right)$ with $K=\left(K_{x} \times K_{\xi}\right) \in \mathcal{T}_{h}$, and for any $\mathbf{U} \in\left[P^{m}\left(K_{x}\right)\right]^{d_{x}}$, the following hold:

$$
\begin{array}{cl}
\left\|\nabla_{\mathbf{x}} g\right\|_{0, K} \leq C h_{K_{x}}^{-1}\|g\|_{0, K}, & \left\|\nabla_{\xi} g\right\|_{0, K} \leq C h_{K_{\xi}}^{-1}\|g\|_{0, K}, \\
\|\mathbf{U}\|_{0, \infty, K_{x}} \leq C h_{K_{x}}^{-d_{x} / 2}\|\mathbf{U}\|_{0, K_{x}}, & \|\mathbf{U}\|_{0, \partial K_{x}} \leq C h_{K_{x}}^{-1 / 2}\|\mathbf{U}\|_{0, K_{x}},
\end{array}
$$

where the constant $C$ is independent of the mesh sizes $h_{K_{x}}, h_{K_{\xi}}$, but depends on $m$ and the shape regularity parameters $\sigma_{x}$ and $\sigma_{\xi}$ of the mesh.

2.2. The Semi-Discrete DG Methods. On the PDE level, the two equations in $1.1 \mathrm{c}$ involving the divergence of the magnetic and electric fields can be derived from the remaining part of the VM system; therefore, the numerical methods proposed in this section are formulated for the VM system without (1.1c). We want to stress that even though in principle the initial satisfaction of these constraints is sufficient for their satisfaction for all time, in certain circumstance one may need to consider explicitly such divergence conditions in order to produce physically relevant numerical simulations [46] ?].

Given $k, r \geq 0$, the semi-discrete DG methods for the VM system are defined by the following procedure: for any $K=K_{x} \times K_{\xi} \in \mathcal{T}_{h}$, look for $f_{h} \in \mathcal{G}_{h}^{k}, \mathbf{E}_{h}, \mathbf{B}_{h} \in \mathcal{U}_{h}^{r}$, such that for any $g \in \mathcal{G}_{h}^{k}, \mathbf{U}, \mathbf{V} \in \mathcal{U}_{h}^{r}$,

$$
\begin{aligned}
& \int_{K} \partial_{t} f_{h} g d \mathbf{x} d \xi-\int_{K} f_{h} \xi \cdot \nabla_{\mathbf{x}} g d \mathbf{x} d \xi-\int_{K} f_{h}\left(\mathbf{E}_{h}+\xi \times \mathbf{B}_{h}\right) \cdot \nabla_{\xi} g d \mathbf{x} d \xi \\
&+\int_{K_{\xi}} \int_{\partial K_{x}} \widehat{f_{h} \xi \cdot \mathbf{n}_{x}} g d s_{x} d \xi+\int_{K_{x}} \int_{\partial K_{\xi}}\left(f_{h}\left(\mathbf{E}_{h} \widehat{\widehat{\xi \times}} \mathbf{B}_{h}\right) \cdot \mathbf{n}_{\xi}\right) g d s_{\xi} d x=0 \\
& \int_{K_{x}} \partial_{t} \mathbf{E}_{h} \cdot \mathbf{U} d \mathbf{x}=\int_{K_{x}} \mathbf{B}_{h} \cdot \nabla \times \mathbf{U} d \mathbf{x}+\int_{\partial K_{x}} \widehat{\mathbf{n}_{x} \times \mathbf{B}_{h}} \cdot \mathbf{U} d s_{x}-\int_{K_{x}} \mathbf{J}_{h} \cdot \mathbf{U} d \mathbf{x} \\
& \int_{K_{x}} \partial_{t} \mathbf{B}_{h} \cdot \mathbf{V} d \mathbf{x}=-\int_{K_{x}} \mathbf{E}_{h} \cdot \nabla \times \mathbf{V} d \mathbf{x}-\int_{\partial K_{x}} \widehat{\mathbf{n}_{x} \times \mathbf{E}_{h}} \cdot \mathbf{V} d s_{x}
\end{aligned}
$$


with

$$
\mathbf{J}_{h}(\mathbf{x}, t)=\int_{\mathcal{T}_{h}^{\xi}} f_{h}(\mathbf{x}, \xi, t) \xi d \xi .
$$

Here $\mathbf{n}_{x}$ and $\mathbf{n}_{\xi}$ are outward unit normals of $\partial K_{x}$ and $\partial K_{\xi}$, respectively. All 'hat' functions are numerical fluxes that are determined by upwinding, i.e.,

$$
\begin{aligned}
& \widehat{f_{h} \xi \cdot \mathbf{n}_{x}}:=\widetilde{f_{h} \xi} \cdot \mathbf{n}_{x}=\left(\left\{f_{h} \xi\right\}_{x}+\frac{\left|\xi \cdot \mathbf{n}_{x}\right|}{2}\left[f_{h}\right]_{x}\right) \cdot \mathbf{n}_{x},
\end{aligned}
$$

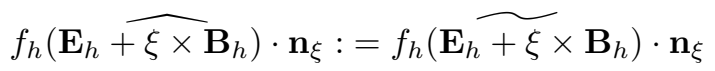

$$
\begin{aligned}
& =\left(\left\{f_{h}\left(\mathbf{E}_{h}+\xi \times \mathbf{B}_{h}\right)\right\}_{\xi}+\frac{\left|\left(\mathbf{E}_{h}+\xi \times \mathbf{B}_{h}\right) \cdot \mathbf{n}_{\xi}\right|}{2}\left[f_{h}\right]_{\xi}\right) \cdot \mathbf{n}_{\xi}, \\
& \widehat{\mathbf{n}_{x} \times \mathbf{E}_{h}}:=\mathbf{n}_{x} \times \widetilde{\mathbf{E}_{h}}=\mathbf{n}_{x} \times\left(\left\{\mathbf{E}_{h}\right\}_{x}+\frac{1}{2}\left[\mathbf{B}_{h}\right]_{\tau}\right), \\
& \widehat{\mathbf{n}_{x} \times \mathbf{B}_{h}}:=\mathbf{n}_{x} \times \widetilde{\mathbf{B}_{h}}=\mathbf{n}_{x} \times\left(\left\{\mathbf{B}_{h}\right\}_{x}-\frac{1}{2}\left[\mathbf{E}_{h}\right]_{\tau}\right),
\end{aligned}
$$

where these relations define the meaning of 'tilde'.

For the Maxwell part, we also consider two other numerical fluxes: central flux and alternating flux, which are defined by

$$
\begin{aligned}
& \text { Central flux: } \widetilde{\mathbf{E}_{h}}=\left\{\mathbf{E}_{h}\right\}, \widetilde{\mathbf{B}_{h}}=\left\{\mathbf{B}_{h}\right\}, \\
& \text { Alternating flux: } \widetilde{\mathbf{E}_{h}}=\mathbf{E}_{h}^{+}, \widetilde{\mathbf{B}_{h}}=\mathbf{B}_{h}^{-}, \text {or } \widetilde{\mathbf{E}_{h}}=\mathbf{E}_{h}^{-}, \widetilde{\mathbf{B}_{h}}=\mathbf{B}_{h}^{+} .
\end{aligned}
$$

Upon summing up 2.6a with respect to $K \in \mathcal{T}_{h}$ and similarly summing $(2.6 \mathrm{~b})$ and $(2.6 \mathrm{c})$ with respect to $K_{x} \in \mathcal{T}_{h}^{x}$, the numerical method becomes the following: look for $f_{h} \in \mathcal{G}_{h}^{k}, \mathbf{E}_{h}, \mathbf{B}_{h} \in \mathcal{U}_{h}^{r}$, such that

$$
\begin{aligned}
a_{h}\left(f_{h}, \mathbf{E}_{h}, \mathbf{B}_{h} ; g\right) & =0, \\
b_{h}\left(\mathbf{E}_{h}, \mathbf{B}_{h} ; \mathbf{U}, \mathbf{V}\right) & =l_{h}\left(\mathbf{J}_{h} ; \mathbf{U}\right),
\end{aligned}
$$

for any $g \in \mathcal{G}_{h}^{k}, \mathbf{U}, \mathbf{V} \in \mathcal{U}_{h}^{r}$, where

$$
\begin{aligned}
a_{h}\left(f_{h}, \mathbf{E}_{h}, \mathbf{B}_{h} ; g\right)= & a_{h, 1}\left(f_{h} ; g\right)+a_{h, 2}\left(f_{h}, \mathbf{E}_{h}, \mathbf{B}_{h} ; g\right), \quad l_{h}\left(\mathbf{J}_{h} ; \mathbf{U}\right)=-\int_{\mathcal{T}_{h}^{x}} \mathbf{J}_{h} \cdot \mathbf{U} d \mathbf{x} \\
b_{h}\left(\mathbf{E}_{h}, \mathbf{B}_{h} ; \mathbf{U}, \mathbf{V}\right)= & \int_{\mathcal{T}_{h}^{x}} \partial_{t} \mathbf{E}_{h} \cdot \mathbf{U} d \mathbf{x}-\int_{\mathcal{T}_{h}^{x}} \mathbf{B}_{h} \cdot \nabla \times \mathbf{U} d \mathbf{x}-\int_{\mathcal{E}_{x}} \widetilde{\mathbf{B}_{h}} \cdot[\mathbf{U}]_{\tau} d s_{x} \\
& +\int_{\mathcal{T}_{h}^{x}} \partial_{t} \mathbf{B}_{h} \cdot \mathbf{V} d \mathbf{x}+\int_{\mathcal{T}_{h}^{x}} \mathbf{E}_{h} \cdot \nabla \times \mathbf{V} d \mathbf{x}+\int_{\mathcal{E}_{x}} \widetilde{\mathbf{E}_{h}} \cdot[\mathbf{V}]_{\tau} d s_{x},
\end{aligned}
$$

and

$$
\begin{gathered}
a_{h, 1}\left(f_{h} ; g\right)=\int_{\mathcal{T}_{h}} \partial_{t} f_{h} g d \mathbf{x} d \xi-\int_{\mathcal{T}_{h}} f_{h} \xi \cdot \nabla_{\mathbf{x}} g d \mathbf{x} d \xi+\int_{\mathcal{T}_{h}^{\xi}} \int_{\mathcal{E}_{x}} \widetilde{f_{h} \xi} \cdot[g]_{x} d s_{x} d \xi \\
a_{h, 2}\left(f_{h}, \mathbf{E}_{h}, \mathbf{B}_{h} ; g\right)=-\int_{\mathcal{T}_{h}} f_{h}\left(\mathbf{E}_{h}+\xi \times \mathbf{B}_{h}\right) \cdot \nabla_{\xi} g d \mathbf{x} d \xi+\int_{\mathcal{T}_{h}^{x}} \int_{\mathcal{E}_{\xi}} f_{h}\left(\mathbf{E}_{h} \widetilde{+\xi} \times \mathbf{B}_{h}\right) \cdot[g]_{\xi} d s_{\xi} d \mathbf{x} .
\end{gathered}
$$

Note, $a_{h}$ is linear with respect to $f_{h}$ and $g$, yet it is in general nonlinear with respect to $\mathbf{E}_{h}$ and $\mathbf{B}_{h}$ due to (2.8b). Recall, the exact solution $f$ has compact support in $\xi$; therefore, the numerical fluxes of (2.8a)-(2.8d) and (2.9a) and (2.9b) are consistent and, consequently, so is the proposed numerical method. That is, the exact solution $(f, \mathbf{E}, \mathbf{B})$ satisfies

$$
\begin{aligned}
a_{h}(f, \mathbf{E}, \mathbf{B} ; g) & =0, \quad \forall g \in \mathcal{G}_{h}^{k}, \\
b_{h}(\mathbf{E}, \mathbf{B} ; \mathbf{U}, \mathbf{V}) & =l_{h}(\mathbf{J} ; \mathbf{U}), \quad \forall \mathbf{U}, \mathbf{V} \in \mathcal{U}_{h}^{r} .
\end{aligned}
$$


2.3. Temporal Discretizations. We use total variation diminishing (TVD) high-order Runge-Kutta methods to solve the method of lines ODE resulting from the semi-discrete DG scheme, $\frac{d}{d t} G_{h}=R\left(G_{h}\right)$. Such time stepping methods are convex combinations of the Euler forward time discretization. The commonly used third-order TVD Runge-Kutta method is given by

$$
\begin{aligned}
G_{h}^{(1)} & =G_{h}^{n}+\triangle t R\left(G_{h}^{n}\right) \\
G_{h}^{(2)} & =\frac{3}{4} G_{h}^{n}+\frac{1}{4} G_{h}^{(1)}+\frac{1}{4} \triangle t R\left(G_{h}^{(1)}\right) \\
G_{h}^{n+1} & =\frac{1}{3} G_{h}^{n}+\frac{2}{3} G_{h}^{(2)}+\frac{2}{3} \triangle t R\left(G_{h}^{(2)}\right),
\end{aligned}
$$

where $G_{h}^{n}$ represents a numerical approximation of the solution at discrete time $t_{n}$. A detailed description of the TVD Runge-Kutta method can be found in [53]; see also [31] and [32] for strong-stability-perserving methods.

3. Conservation and Stability. In this section, we will establish conservation and stability properties of the semi-discrete DG methods. In particular, we prove that subject to boundary effects, the total charge (mass) is always conserved. As for the total energy of the system, conservation depends on the choice of numerical fluxes for the Maxwell equations. We also show that $f_{h}$ is $L^{2}$ stable, which facilitates the error analysis of Section 4.

Lemma 3.1 (Mass conservation). The numerical solution $f_{h} \in \mathcal{G}_{h}^{k}$ with $k \geq 0$ satisfies

$$
\frac{d}{d t} \int_{\mathcal{T}_{h}} f_{h} d \mathbf{x} d \xi+\Theta_{h, 1}(t)=0
$$

where

$$
\Theta_{h, 1}(t)=\int_{\mathcal{T}_{h}^{x}} \int_{\mathcal{E}_{\xi}^{b}} f_{h} \max \left(\left(\mathbf{E}_{h}+\xi \times \mathbf{B}_{h}\right) \cdot \mathbf{n}_{\xi}, 0\right) d s_{\xi} d \mathbf{x}
$$

Equivalently, with $\rho_{h}(\mathbf{x}, t)=\int_{\mathcal{T}_{h}^{\xi}} f_{h}(\mathbf{x}, \xi, t) d \xi$, for any $T>0$, the following holds:

$$
\int_{\mathcal{T}_{h}^{x}} \rho_{h}(\mathbf{x}, T) d \mathbf{x}+\int_{0}^{T} \Theta_{h, 1}(t) d t=\int_{\mathcal{T}_{h}^{x}} \rho_{h}(\mathbf{x}, 0) d \mathbf{x}
$$

Proof. Let $g(\mathbf{x}, \xi)=1$. Note that $g \in \mathcal{G}_{h}^{k}$, for any $k \geq 0$, is continuous and $\nabla_{\mathbf{x}} g=0$. Taking this $g$ as the test function in 2.10a), one has

$$
\frac{d}{d t} \int_{\mathcal{T}_{h}} f_{h} d \mathbf{x} d \xi+\int_{\mathcal{T}_{h}^{x}} \int_{\mathcal{E}_{\xi}^{b}} f_{h}\left(\mathbf{E}_{h} \widetilde{+\xi} \times \mathbf{B}_{h}\right) \cdot[g]_{\xi} d s_{\xi} d \mathbf{x}=0 .
$$

With the numerical flux of 2.8b and the average and jump across $\mathcal{E}_{\xi}^{b}$ of 2.4, the second term above becomes

$$
\begin{aligned}
& \int_{\mathcal{T}_{h}^{x}} \int_{\mathcal{E}_{\xi}^{b}} f_{h}\left(\mathbf{E}_{h} \widetilde{+\xi} \times \mathbf{B}_{h}\right) \cdot \mathbf{n}_{\xi} d s_{\xi} d \mathbf{x} \\
= & \int_{\mathcal{T}_{h}^{x}} \int_{\mathcal{E}_{\xi}^{b}} \frac{f_{h}}{2}\left(\left(\mathbf{E}_{h}+\xi \times \mathbf{B}_{h}\right) \cdot \mathbf{n}_{\xi}+\left|\left(\mathbf{E}_{h}+\xi \times \mathbf{B}_{h}\right) \cdot \mathbf{n}_{\xi}\right|\right) d s_{\xi} d \mathbf{x}=\Theta_{h, 1}(t),
\end{aligned}
$$

and this gives (3.1). Integrating in time from 0 to $T$ gives 3.2 .

LEMma 3.2 (Energy conservation 1). For $k \geq 2, r \geq 0$, the numerical solution $f_{h} \in \mathcal{G}_{h}^{k}, \mathbf{E}_{h}, \mathbf{B}_{h} \in \mathcal{U}_{h}^{r}$ with the upwind numerical fluxes (2.8a)-2.8d satisfies

$$
\frac{d}{d t}\left(\int_{\mathcal{T}_{h}} f_{h}|\xi|^{2} d \mathbf{x} d \xi+\int_{\mathcal{T}_{h}^{x}}\left(\left|\mathbf{E}_{h}\right|^{2}+\left|\mathbf{B}_{h}\right|^{2}\right) d \mathbf{x}\right)+\Theta_{h, 2}(t)+\Theta_{h, 3}(t)=0
$$


with

$$
\Theta_{h, 2}(t)=\int_{\mathcal{E}_{x}}\left(\left|\left[\mathbf{E}_{h}\right]_{\tau}\right|^{2}+\left|\left[\mathbf{B}_{h}\right]_{\tau}\right|^{2}\right) d s_{x}, \quad \Theta_{h, 3}(t)=\int_{\mathcal{T}_{h}^{x}} \int_{\mathcal{E}_{\xi}^{b}} f_{h}|\xi|^{2} \max \left(\left(\mathbf{E}_{h}+\xi \times \mathbf{B}_{h}\right) \cdot \mathbf{n}_{\xi}, 0\right) d s_{\xi} d \mathbf{x}
$$

Proof. Step 1: Let $g(\mathbf{x}, \xi)=|\xi|^{2}$. Note that $g \in \mathcal{G}_{h}^{k}$ for $k \geq 2$ and it is continuous. In addition, $\nabla_{\mathbf{x}} g=0, \nabla_{\xi} g=2 \xi$, and $\xi \times \mathbf{U} \cdot \nabla_{\xi} g=0$ for any function $\mathbf{U}$. Taking this $g$ as the test function in (2.10a), one has

$$
\begin{aligned}
& \frac{d}{d t} \int_{\mathcal{T}_{h}} f_{h}|\xi|^{2} d \mathbf{x} d \xi=2 \int_{\mathcal{T}_{h}} f_{h} \mathbf{E}_{h} \cdot \xi d \mathbf{x} d \xi-\int_{\mathcal{T}_{h}^{x}} \int_{\mathcal{E}_{\xi}^{b}} f_{h}\left(\mathbf{E}_{h} \widetilde{+\xi} \times \mathbf{B}_{h}\right) \cdot\left[|\xi|^{2}\right]_{\xi} d s_{\xi} d \mathbf{x} \\
& =2 \int_{\mathcal{T}_{h}^{x}} \mathbf{E}_{h} \cdot\left(\int_{\mathcal{T}_{h}^{\xi}} f_{h} \xi d \xi\right) d \mathbf{x}-\int_{\mathcal{T}_{h}^{x}} \int_{\mathcal{E}_{\xi}^{b}}\left(\frac{1}{2}\left(\mathbf{E}_{h}+\xi \times \mathbf{B}_{h}\right) f_{h}+\frac{\left|\left(\mathbf{E}_{h}+\xi \times \mathbf{B}_{h}\right) \cdot \mathbf{n}_{\xi}\right|}{2} f_{h} \mathbf{n}_{\xi}\right) \cdot\left(|\xi|^{2} \mathbf{n}_{\xi}\right) d s_{\xi} d \mathbf{x} \\
& =2 \int_{\mathcal{T}_{h}^{x}} \mathbf{E}_{h} \cdot \mathbf{J}_{h} d \mathbf{x}-\int_{\mathcal{T}_{h}^{x}} \int_{\mathcal{E}_{\xi}^{b}} \frac{f_{h}}{2}\left(\left(\mathbf{E}_{h}+\xi \times \mathbf{B}_{h}\right) \cdot \mathbf{n}_{\xi}+\left|\left(\mathbf{E}_{h}+\xi \times \mathbf{B}_{h}\right) \cdot \mathbf{n}_{\xi}\right|\right)|\xi|^{2} d s_{\xi} d \mathbf{x} \\
& =2 \int_{\mathcal{T}_{h}^{x}} \mathbf{E}_{h} \cdot \mathbf{J}_{h} d \mathbf{x}-\int_{\mathcal{T}_{h}^{x}} \int_{\mathcal{E}_{\xi}^{b}} f_{h}|\xi|^{2} \max \left(\left(\mathbf{E}_{h}+\xi \times \mathbf{B}_{h}\right) \cdot \mathbf{n}_{\xi}, 0\right) d s_{\xi} d \mathbf{x}
\end{aligned}
$$

Step 2: With $\mathbf{U}=\mathbf{E}_{h}$ and $\mathbf{V}=\mathbf{B}_{h}, 2.10 \mathrm{~b}$ becomes

$$
\begin{aligned}
-\int_{\mathcal{T}_{h}^{x}} \mathbf{J}_{h} \cdot \mathbf{E}_{h} d \mathbf{x} & =\frac{1}{2} \frac{d}{d t} \int_{\mathcal{T}_{h}^{x}}\left|\mathbf{E}_{h}\right|^{2} d \mathbf{x}-\int_{\mathcal{T}_{h}^{x}} \mathbf{B}_{h} \cdot \nabla \times \mathbf{E}_{h} d \mathbf{x}-\int_{\mathcal{E}_{x}} \widetilde{\mathbf{B}_{h}} \cdot\left[\mathbf{E}_{h}\right]_{\tau} d s_{x} \\
& +\frac{1}{2} \frac{d}{d t} \int_{\mathcal{T}_{h}^{x}}\left|\mathbf{B}_{h}\right|^{2} d \mathbf{x}+\int_{\mathcal{T}_{h}^{x}} \mathbf{E}_{h} \cdot \nabla \times \mathbf{B}_{h} d \mathbf{x}+\int_{\mathcal{E}_{x}} \widetilde{\mathbf{E}_{h}} \cdot\left[\mathbf{B}_{h}\right]_{\tau} d s_{x}, \\
& =\frac{1}{2} \frac{d}{d t} \int_{\mathcal{T}_{h}^{x}}\left(\left|\mathbf{E}_{h}\right|^{2}+\left|\mathbf{B}_{h}\right|^{2}\right) d \mathbf{x}-\int_{\mathcal{E}_{x}}\left(\left[\mathbf{E}_{h} \times \mathbf{B}_{h}\right]_{x}+\widetilde{\mathbf{B}_{h}} \cdot\left[\mathbf{E}_{h}\right]_{\tau}-\widetilde{\mathbf{E}_{h}} \cdot\left[\mathbf{B}_{h}\right]_{\tau}\right) d s_{x}, \\
& =\frac{1}{2} \frac{d}{d t} \int_{\mathcal{T}_{h}^{x}}\left(\left|\mathbf{E}_{h}\right|^{2}+\left|\mathbf{B}_{h}\right|^{2}\right) d \mathbf{x}+\frac{1}{2} \int_{\mathcal{E}_{x}}\left(\left|\left[\mathbf{E}_{h}\right]_{\tau}\right|^{2}+\left|\left[\mathbf{B}_{h}\right]_{\tau}\right|^{2}\right) d s_{x} .
\end{aligned}
$$

The last equality uses the formulas of the upwind fluxes $(2.8 \mathrm{c})-(2.8 \mathrm{~d})$ as well as $(2.5 \mathrm{~b})$.

Combining the results in previous two steps, one concludes (3.5).

Corollary 3.3 (Energy conservation 2). For $k \geq 2, r \geq 0$ and the numerical solution $f_{h} \in \mathcal{G}_{h}^{k}$, $\mathbf{E}_{h}, \mathbf{B}_{h} \in \mathcal{U}_{h}^{r}$ with the upwind numerical flux 2.8a)-2.8b for the Vlasov part, and with either the central or alternating flux of 2.9a)-2.9b for the Maxwell part, the following holds:

$$
\frac{d}{d t}\left(\int_{\mathcal{T}_{h}} f_{h}|\xi|^{2} d \mathbf{x} d \xi+\int_{\mathcal{T}_{h}^{x}}\left(\left|\mathbf{E}_{h}\right|^{2}+\left|\mathbf{B}_{h}\right|^{2}\right) d \mathbf{x}\right)+\Theta_{h, 3}(t)=0 .
$$

Proof. The proof proceeds the same way as for Lemma 3.2 . The only difference is that here the equalities (2.5b)-2.5c) give

$$
\left[\mathbf{E}_{h} \times \mathbf{B}_{h}\right]_{x}+\widetilde{\mathbf{B}_{h}} \cdot\left[\mathbf{E}_{h}\right]_{\tau}-\widetilde{\mathbf{E}_{h}} \cdot\left[\mathbf{B}_{h}\right]_{\tau}=0,
$$

which holds for $\widetilde{\mathbf{E}_{h}}$ and $\widetilde{\mathbf{B}_{h}}$ defined in the central or alternating flux in the Maxwell solver.

With either the central or alternating flux for the Maxwell solver, the energy does not change due to the tangential jump of the magnetic and electric fields as in Lemma 3.2 . This, on the other hand, may have some effect on the accuracy of the methods (See Sections 4 and 5 and also [1]).

REMARK 3.4. In Lemma 3.2, the conservation error term satisfies $\Theta_{h, 2} \geq 0$ with equality depending on the choice of numerical fluxes for the Maxwell discretization. In addition, the error conservation terms $\Theta_{h, 1}$ in Lemma 3.1 and $\Theta_{h, 3}$ in Lemmas 3.2 and Corollary 3.3 both depend on the numerical solution $f_{h}$ on the outflow portion of the computational boundary in $\xi$-space, which is determined by the numerical electric 
and magnetic fields. Hence, for the case of periodic boundary conditions in $x$-space for both the Vlasov and Maxwell's equations, it can be easily shown that these error terms $\Theta_{h, i} \approx 0$, for $i=1$ and 3 , by choosing the computational domain in $\xi$-space sufficiently large.

REMARK 3.5. Energy conservation holds as long as $|\xi|^{2} \in \mathcal{G}_{h}^{k}$. Indeed, for $k<2$, the energy conservation results of Lemma 3.2 and Corollary 3.3 can be obtained if one replaces $\mathcal{G}_{h}^{k}$ with $\tilde{\mathcal{G}}_{h}^{k}=\mathcal{G}_{h}^{k} \oplus\left\{|\xi|^{2}\right\}=\{g+$ $\left.c|\xi|^{2}, \forall g \in \mathcal{G}_{h}^{k}, \forall c \in \mathbb{R}\right\}$.

Finally, we can obtain the $L^{2}$-stability result for $f_{h}$, a result that is independent of choice of numerical flux in the Maxwell solver. This result will be used in the error analysis of Section 4.

Lemma $3.6\left(L^{2}\right.$-stability of $\left.f_{h}\right)$. For $k \geq 0$, the numerical solution $f_{h} \in \mathcal{G}_{h}^{k}$ satisfies

$$
\begin{aligned}
\frac{d}{d t}\left(\int_{\mathcal{T}_{h}}\left|f_{h}\right|^{2} d \mathbf{x} d \xi\right) & +\int_{\mathcal{T}_{h}^{\xi}} \int_{\mathcal{E}_{x}}\left|\xi \cdot \mathbf{n}_{x}\right|\left|\left[f_{h}\right]_{x}\right|^{2} d s_{x} d \xi \\
& +\int_{\mathcal{T}_{h}^{x}} \int_{\mathcal{E}_{\xi}}\left|\left(\mathbf{E}_{h}+\xi \times \mathbf{B}_{h}\right) \cdot \mathbf{n}_{\xi}\right|\left|\left[f_{h}\right]_{\xi}\right|^{2} d s_{\xi} d \mathbf{x}=0 .
\end{aligned}
$$

Proof. Taking $g=f_{h}$ in 2.10a), one gets

$$
\frac{1}{2} \frac{d}{d t}\left(\int_{\mathcal{T}_{h}}\left|f_{h}\right|^{2} d \mathbf{x} d \xi\right)+R_{1}+R_{2}=0
$$

with

$$
R_{1}=-\int_{\mathcal{T}_{h}} f_{h} \xi \cdot \nabla_{\mathbf{x}} f_{h} d \mathbf{x} d \xi+\int_{\mathcal{T}_{h}^{\xi}} \int_{\mathcal{E}_{x}} \widetilde{f_{h} \xi} \cdot\left[f_{h}\right]_{x} d s_{x} d \xi, \quad R_{2}=a_{h, 2}\left(f_{h}, \mathbf{E}_{h}, \mathbf{B}_{h} ; f_{h}\right) .
$$

Observe

$$
\begin{aligned}
R_{1} & =-\int_{\mathcal{T}_{h}^{\xi}} \sum_{K_{x} \in \mathcal{T}_{h}^{x}} \int_{K_{x}} \xi \cdot \nabla_{\mathbf{x}}\left(\frac{f_{h}^{2}}{2}\right) d \mathbf{x} d \xi+\int_{\mathcal{T}_{h}^{\xi}} \int_{\mathcal{E}_{x}} \widetilde{f_{h} \xi} \cdot\left[f_{h}\right]_{x} d s_{x} d \xi, \\
& =-\int_{\mathcal{T}_{h}^{\xi}} \sum_{K_{x} \in \mathcal{T}_{h}^{x}} \int_{\partial K_{x}} \xi \cdot \mathbf{n}_{x}\left(\frac{f_{h}^{2}}{2}\right) d s_{x} d \xi+\int_{\mathcal{T}_{h}^{\xi}} \int_{\mathcal{E}_{x}} \widetilde{f_{h} \xi} \cdot\left[f_{h}\right]_{x} d s_{x} d \xi, \\
& =-\int_{\mathcal{T}_{h}^{\xi}} \int_{\mathcal{E}_{x}} \frac{1}{2}\left[\xi f_{h}^{2}\right]_{x} d s_{x} d \xi+\int_{\mathcal{T}_{h}^{\xi}} \int_{\mathcal{E}_{x}} \widetilde{f_{h} \xi} \cdot\left[f_{h}\right]_{x} d s_{x} d \xi, \\
& =\int_{\mathcal{T}_{h}^{\xi}} \int_{\mathcal{E}_{x}}\left(-\frac{1}{2}\left[\xi f_{h}^{2}\right]_{x}+\left\{f_{h} \xi\right\}_{x} \cdot\left[f_{h}\right]_{x}+\frac{1}{2}\left|\xi \cdot \mathbf{n}_{x}\right|\left[f_{h}\right]_{x} \cdot\left[f_{h}\right]_{x}\right) d s_{x} d \xi, \\
& =\int_{\mathcal{T}_{h}^{\xi}} \int_{\mathcal{E}_{x}}\left(\left(-\frac{1}{2}\left[f_{h}^{2}\right]_{x}+\left\{f_{h}\right\}_{x}\left[f_{h}\right]_{x}\right) \cdot \xi+\frac{1}{2}\left|\xi \cdot \mathbf{n}_{x}\right|\left|\left[f_{h}\right]_{x}\right|^{2}\right) d s_{x} d \xi, \\
& =\frac{1}{2} \int_{\mathcal{T}_{h}^{\xi}} \int_{\mathcal{E}_{x}}\left|\xi \cdot \mathbf{n}_{x}\right|\left|\left[f_{h}\right]_{x}\right|^{2} d s_{x} d \xi,
\end{aligned}
$$

where the fourth equality uses the definition of the numerical flux 2.8a) and the last one is due to 2.5a). Similarly,

$$
\begin{aligned}
R_{2} & =-\int_{\mathcal{T}_{h}^{x}} \sum_{K_{\xi} \in \mathcal{T}_{h}^{\xi}} \int_{K_{\xi}}\left(\mathbf{E}_{h}+\xi \times \mathbf{B}_{h}\right) \cdot \nabla_{\xi}\left(\frac{f_{h}^{2}}{2}\right) d \xi d \mathbf{x}+\int_{\mathcal{T}_{h}^{x}} \int_{\mathcal{E}_{\xi}} f_{h}\left(\mathbf{E}_{h} \widetilde{+\xi} \times \mathbf{B}_{h}\right) \cdot\left[f_{h}\right]_{\xi} d s_{\xi} d \mathbf{x}, \\
& =\int_{\mathcal{T}_{h}^{x}} \int_{\mathcal{E}_{\xi}}\left(-\frac{1}{2}\left[\left(\mathbf{E}_{h}+\xi \times \mathbf{B}_{h}\right) f_{h}^{2}\right]_{\xi}+\left\{f_{h}\left(\mathbf{E}_{h}+\xi \times \mathbf{B}_{h}\right)\right\}_{\xi} \cdot\left[f_{h}\right]_{\xi}+\frac{1}{2}\left|\left(\mathbf{E}_{h}+\xi \times \mathbf{B}_{h}\right) \cdot \mathbf{n}_{\xi}\right|\left[f_{h}\right]_{\xi} \cdot\left[f_{h}\right]_{\xi}\right) d s_{\xi} d \mathbf{x}, \\
& =\int_{\mathcal{T}_{h}^{x}} \int_{\mathcal{E}_{\xi}}\left(\left(-\frac{1}{2}\left[f_{h}^{2}\right]_{\xi}+\left\{f_{h}\right\}_{\xi} \cdot\left[f_{h}\right]_{\xi}\right) \cdot\left(\mathbf{E}_{h}+\xi \times \mathbf{B}_{h}\right)+\frac{1}{2}\left|\left(\mathbf{E}_{h}+\xi \times \mathbf{B}_{h}\right) \cdot \mathbf{n}_{\xi}\right|\left|\left[f_{h}\right]_{\xi}\right|^{2}\right) d s_{\xi} d \mathbf{x}, \\
& =\frac{1}{2} \int_{\mathcal{T}_{h}^{x}} \int_{\mathcal{E}_{\xi}}\left|\left(\mathbf{E}_{h}+\xi \times \mathbf{B}_{h}\right) \cdot \mathbf{n}_{\xi}\right|\left|\left[f_{h}\right]_{\xi}\right|^{2} d s_{\xi} d \mathbf{x},
\end{aligned}
$$


where the second equality is due to $\nabla_{\xi} \cdot\left(\mathbf{E}_{h}+\xi \times \mathbf{B}_{h}\right)=0$ and the definition of the numerical flux in (2.8b), and the third equality uses (2.5a) and $\mathbf{E}_{h}+\xi \times \mathbf{B}_{h}$ being continuous in $\xi$. With (3.7), we conclude $L^{2}$ stability (3.6).

4. Error Estimates. In this section, we establish error estimates at any given time $T>0$ for our semi-discrete DG methods described in Section 2.2. It is assumed that the discrete spaces have the same degree, i.e., $k=r$, and that the exact solution satisfies $f \in C^{1}\left([0, T] ; H^{k+1}(\Omega) \cap W^{1, \infty}(\Omega)\right)$ and $\mathbf{E}, \mathbf{B} \in$ $C^{0}\left([0, T] ;\left[H^{k+1}\left(\Omega_{x}\right)\right]^{d_{x}} \cap\left[W^{1, \infty}\left(\Omega_{x}\right)\right]^{d_{x}}\right)$. Also, periodic boundary conditions in $\mathbf{x}$ and compact support for $f$ in $\xi$ are assumed. To prevent the proliferation of constants, we use $A \lesssim B$ to represent the inequality $A \leq$ (constant) $B$, where the positive constant is independent of the mesh size $h, h_{x}$, and $h_{\xi}$, but it can depend on the polynomial degree $k$, mesh parameters $\sigma_{0}, \sigma_{x}$ and $\sigma_{\xi}$, and domain parameters $L_{x}$ and $L_{\xi}$.

Defining $\zeta_{h}=\Pi^{k} f-f$ and $\varepsilon_{h}=\Pi^{k} f-f_{h}$, it follows that $f-f_{h}=\varepsilon_{h}-\zeta_{h}$. Analogously, if $\boldsymbol{\zeta}_{h}^{E}=\Pi_{x}^{k} \mathbf{E}-\mathbf{E}$, $\boldsymbol{\zeta}_{h}^{B}=\boldsymbol{\Pi}_{x}^{k} \mathbf{B}-\mathbf{B}, \varepsilon_{h}^{E}=\boldsymbol{\Pi}_{x}^{k} \mathbf{E}-\mathbf{E}_{h}$ and $\varepsilon_{h}^{B}=\boldsymbol{\Pi}_{x}^{k} \mathbf{B}-\mathbf{B}_{h}$, then $\mathbf{E}-\mathbf{E}_{h}=\varepsilon_{h}^{E}-\boldsymbol{\zeta}_{h}^{E}$ and $\mathbf{B}-\mathbf{B}_{h}=\varepsilon_{h}^{B}-\boldsymbol{\zeta}_{h}^{B}$. With the approximation results of Lemma 2.1, we have

$$
\left\|\zeta_{h}\right\|_{0, \Omega} \lesssim h^{k+1}|| f\left\|_{k+1, \Omega}, \quad\right\| \boldsymbol{\zeta}_{h}^{B}\left\|_{0, \Omega_{x}} \lesssim h_{x}^{k+1}\right\| \mathbf{B}\left\|_{k+1, \Omega_{x}}, \quad\right\| \boldsymbol{\zeta}_{h}^{E}\left\|_{0, \Omega_{x}} \lesssim h_{x}^{k+1}\right\| \mathbf{E} \|_{k+1, \Omega_{x}} ;
$$

therefore, we only need to estimate $\varepsilon_{h}, \varepsilon_{h}^{E}$ and $\varepsilon_{h}^{B}$. The remainder of this section is organized as follows: we first state Lemmas 4.1 and 4.2 , with which the main error estimate is established in Theorem 4.3 for the proposed semi-discrete DG method with the upwind numerical fluxes. Then, the proofs of Lemmas 4.1 and 4.2 will be given in subsections 4.1 and 4.2 . Lastly, for the proposed method using the central or alternating flux of (2.9a)-2.9b) for the Maxwell solver, error estimates are given in Theorem 4.6 .

LEMMA 4.1 (Estimate of $\varepsilon_{h}$ ). Based on the semi-discrete $D G$ discretization for the Vlasov equation of (2.10a) with the upwind flux (2.8a)-(2.8b), we have

$$
\begin{aligned}
& \frac{d}{d t}\left(\int_{\mathcal{T}_{h}}\left|\varepsilon_{h}\right|^{2} d \mathbf{x} d \xi\right)+\int_{\mathcal{T}_{h}^{\xi}} \int_{\mathcal{E}_{x}}\left|\xi \cdot \mathbf{n}_{x} \|\left[\varepsilon_{h}\right]_{x}\right|^{2} d s_{x} d \xi+\left.\int_{\mathcal{T}_{h}^{x}} \int_{\mathcal{E}_{\xi}}\left(\left|\left(\mathbf{E}_{h}+\xi \times \mathbf{B}_{h}\right) \cdot \mathbf{n}_{\xi}\right|\right)||\left[\varepsilon_{h}\right]_{\xi}\right|^{2} d s_{\xi} d \mathbf{x} \\
& \lesssim\left(h^{k+1} \hat{\Lambda}+h^{k}|| f \|_{k+1, \Omega}\left(\left\|\varepsilon_{h}^{E}\right\|_{0, \infty, \Omega_{x}}+\left\|\varepsilon_{h}^{B}\right\|_{0, \infty, \Omega_{x}}\right)+|f|_{1, \infty, \Omega}\left(\left\|\varepsilon_{h}^{E}\right\|_{0, \Omega_{x}}+\left\|\varepsilon_{h}^{B}\right\| \|_{0, \Omega_{x}}\right)\right)\left\|\varepsilon_{h}\right\|_{0, \Omega} \\
& \quad+h^{k+\frac{1}{2}}\|f\|_{k+1, \Omega}\left(\left\|\varepsilon_{h}^{B}\right\|_{0, \infty, \Omega_{x}}^{1 / 2}+\left\|\varepsilon_{h}^{E}\right\|_{0, \infty, \Omega_{x}}^{1 / 2}+\|\mathbf{B}\|_{0, \infty, \Omega_{x}}^{1 / 2}+\|\mathbf{E}\|_{0, \infty, \Omega_{x}}^{1 / 2}\right) \\
& \quad \times\left(\int_{\mathcal{T}_{h}^{x}} \int_{\mathcal{E}_{\xi}}\left|\left(\mathbf{E}_{h}+\xi \times \mathbf{B}_{h}\right) \cdot \mathbf{n}_{\xi} \|\left[\varepsilon_{h}\right]\right|^{2} d s_{\xi} d x\right)^{1 / 2} \\
& \quad+h^{k+\frac{1}{2}}\|f\|_{k+1, \Omega}\left(\int_{\mathcal{T}_{h}^{\xi}} \int_{\mathcal{E}_{x}}\left|\xi \cdot \mathbf{n}_{x} \|\left[\varepsilon_{h}\right]_{x}\right|^{2} d s_{x} d \xi\right)^{1 / 2}
\end{aligned}
$$

with

$$
\begin{aligned}
\hat{\Lambda}=\left\|\partial_{t} f\right\|_{k+1, \Omega} & +\left(1+\|\mathbf{E}\|_{1, \infty, \Omega_{x}}+\|\mathbf{B}\|_{1, \infty, \Omega_{x}}\right)\|f\|_{k+1, \Omega} \\
& +\left(\|\mathbf{E}\|_{k+1, \Omega_{x}}+\|\mathbf{B}\|_{k+1, \Omega_{x}}\right)|f|_{1, \infty, \Omega} .
\end{aligned}
$$

Lemma 4.2 (Estimate of $\varepsilon_{h}^{E}$ and $\varepsilon_{h}^{B}$ ). Based on the semi-discrete DG discretization for the Maxwell equations of $(2.10 \mathrm{~b})$ with the upwind flux $(2.8 \mathrm{c})-(2.8 \mathrm{~d})$, we have

$$
\begin{aligned}
& \frac{d}{d t} \int_{\mathcal{T}_{h}^{x}}\left(\left|\varepsilon_{h}^{E}\right|^{2}+\left|\varepsilon_{h}^{B}\right|^{2}\right) d \mathbf{x}+\int_{\mathcal{E}_{x}}\left(\left|\left[\varepsilon_{h}^{E}\right]_{\tau}\right|^{2}+\left|\left[\varepsilon_{h}^{B}\right]_{\tau}\right|^{2}\right) d s_{x} \\
& \lesssim\left(\left\|\varepsilon_{h}\right\|_{0, \Omega}+h^{k+1}|| f \|_{k+1, \Omega}\right)\left\|\varepsilon_{h}^{E}\right\|_{0, \Omega_{x}}+h_{x}^{k+\frac{1}{2}}\left(\|\mathbf{E}\|_{k+1, \Omega_{x}}+\left.\|\mathbf{B}\|\right|_{k+1, \Omega_{x}}\right)\left(\int_{\mathcal{E}_{x}}\left|\left[\varepsilon_{h}^{E}\right]_{\tau}\right|^{2}+\left|\left[\varepsilon_{h}^{B}\right]_{\tau}\right|^{2} d s_{x}\right)^{1 / 2} .
\end{aligned}
$$

Theorem 4.3 (Error estimate 1). For $k \geq 2$, the semi-discrete DG method of 2.10a - 2.10b), for the Vlasov-Maxwell equations with the upwind fluxes of (2.8a)-(2.8d), has the following error estimate

$$
\left\|\left(f-f_{h}\right)(t)\right\|_{0, \Omega}^{2}+\left\|\left(\mathbf{E}-\mathbf{E}_{h}\right)(t)\right\|_{0, \Omega_{x}}^{2}+\left\|\left(\mathbf{B}-\mathbf{B}_{h}\right)(t)\right\|_{0, \Omega_{x}}^{2} \leq C h^{2 k+1}, \quad \forall t \in[0, T] .
$$


Here the constant $C$ depends on the upper bounds of $\left\|\left.\partial_{t} f\right|_{k+1, \Omega},\right\| f||_{k+1, \Omega},|f|_{1, \infty, \Omega},\left\|\left.\mathbf{E}\right|_{1, \infty, \Omega_{x}},\right\| \mathbf{B} \|_{1, \infty, \Omega_{x}}$, $\|\mathbf{E}\|_{k+1, \Omega_{x}},\|\mathbf{B}\|_{k+1, \Omega_{x}}$ over the time interval $[0, T]$, and it also depends on the polynomial degree $k$, mesh parameters $\sigma_{0}, \sigma_{x}$ and $\sigma_{\xi}$, and domain parameters $L_{x}$ and $L_{\xi}$.

Proof. With several applications of Cauchy-Schwarz inequality and

$$
\widetilde{\Lambda}=h^{1 / 2} \hat{\Lambda}+\|f\|_{k+1, \Omega}\left(1+\|\mathbf{E}\|_{0, \infty, \Omega_{x}}^{1 / 2}+\|\mathbf{B}\|_{0, \infty, \Omega_{x}}^{1 / 2}\right),
$$

Eq. 4.2 becomes

$$
\begin{aligned}
& \frac{d}{d t}\left(\int_{\mathcal{T}_{h}}\left|\varepsilon_{h}\right|^{2} d \mathbf{x} d \xi\right) \\
& \leq c\left(h^{2 k+1} \widetilde{\Lambda}^{2}+\left(h^{k}\|f\|_{k+1, \Omega}\left(\left\|\varepsilon_{h}^{E}\right\|\left\|_{0, \infty, \Omega_{x}}+\right\| \varepsilon_{h}^{B} \|_{0, \infty, \Omega_{x}}\right)+|f|_{1, \infty, \Omega}\left(\left\|\varepsilon_{h}^{E}\right\|_{0, \Omega_{x}}+\left\|\varepsilon_{h}^{B}\right\| \|_{0, \Omega_{x}}\right)\right)^{2}\right. \\
& \left.\quad+h^{2 k+1}\|f\|_{k+1, \Omega}^{2}\left(\left\|\varepsilon_{h}^{E}\right\|_{0, \infty, \Omega_{x}}+\left\|\varepsilon_{h}^{B}\right\|_{0, \infty, \Omega_{x}}\right)\right)+\left\|\varepsilon_{h}\right\|_{0, \Omega}^{2} \\
& \leq c\left(h^{2 k+1} \widetilde{\Lambda}^{2}+h^{2 k}(1+h)\|f\|_{k+1, \Omega}^{2}\left(\left\|\varepsilon_{h}^{E}\right\|_{0, \infty, \Omega_{x}}^{2}+\left\|\varepsilon_{h}^{B}\right\|_{0, \infty, \Omega_{x}}^{2}\right)+|f|_{1, \infty, \Omega}^{2}\left(\left\|\varepsilon_{h}^{E}\right\|_{0, \Omega_{x}}^{2}+\left\|\varepsilon_{h}^{B}\right\|_{0, \Omega_{x}}^{2}\right)\right) \\
& \quad+\left\|\varepsilon_{h}\right\|_{0, \Omega}^{2} .
\end{aligned}
$$

Here and below, the constant $c>0$ only depends on $k$, mesh parameters $\sigma_{0}, \sigma_{x}$ and $\sigma_{\xi}$, and domain parameters $L_{x}$ and $L_{\xi}$. Moreover, with the inverse inequality of Lemma 2.2 and $\frac{h_{\xi}}{h_{x, \min }}$ being uniformly bounded by $\sigma_{0}$ when the mesh is refined, we have

$$
h^{2 k}\left(\left\|\varepsilon_{h}^{E}\right\|_{0, \infty, \Omega_{x}}^{2}+\left\|\varepsilon_{h}^{B}\right\|_{0, \infty, \Omega_{x}}^{2}\right) \leq c h^{2 k-d_{x}}\left(\left\|\varepsilon_{h}^{E}\right\|_{0, \Omega_{x}}^{2}+\left\|\varepsilon_{h}^{B}\right\|_{0, \Omega_{x}}^{2}\right)
$$

and this leads to

$$
\begin{aligned}
& \frac{d}{d t}\left(\int_{\mathcal{T}_{h}}\left|\varepsilon_{h}\right|^{2} d \mathbf{x} d \xi\right) \\
& \leq c\left(h^{2 k+1} \widetilde{\Lambda}^{2}+\left(h^{2 k-d_{x}}(1+h)\|f\|_{k+1, \Omega}^{2}+|f|_{1, \infty, \Omega}^{2}\right)\left(\left\|\varepsilon_{h}^{E}\right\|_{0, \Omega_{x}}^{2}+\left\|\varepsilon_{h}^{B}\right\|_{0, \Omega_{x}}^{2}\right)\right)+\left\|\varepsilon_{h}\right\|_{0, \Omega}^{2} .
\end{aligned}
$$

Recall $d_{x}=3$, then for $k \geq 2$, there is $2 k-d_{x} \geq 0$ and therefore $h^{2 k-d_{x}}<\infty$. Similarly, with the Cauchy-Schwarz inequality, $\sqrt{4.3}$ becomes

$$
\begin{aligned}
& \frac{d}{d t} \int_{\mathcal{T}_{h}^{x}}\left(\left|\varepsilon_{h}^{E}\right|^{2}+\left|\varepsilon_{h}^{B}\right|^{2}\right) d \mathbf{x} \\
& \leq c\left(\left\|\varepsilon_{h}\right\|_{0, \Omega}^{2}+h^{2 k+2}\|f\|_{k+1, \Omega}^{2}+h_{x}^{2 k+1}\left(\|\mathbf{E}\|_{k+1, \Omega_{x}}^{2}+\|\mathbf{B}\|_{k+1, \Omega_{x}}^{2}\right)\right)+\left\|\varepsilon_{h}^{E}\right\|_{0, \Omega_{x}}^{2} .
\end{aligned}
$$

Now, summing up (4.6) and 4.7), we get

$$
\frac{d}{d t}\left(\int_{\mathcal{T}_{h}}\left|\varepsilon_{h}\right|^{2} d \mathbf{x} d \xi+\int_{\mathcal{T}_{h}^{x}}\left|\varepsilon_{h}^{E}\right|^{2}+\left|\varepsilon_{h}^{B}\right|^{2} d \mathbf{x}\right) \leq \Lambda h^{2 k+1}+\Theta\left(\int_{\mathcal{T}_{h}}\left|\varepsilon_{h}\right|^{2} d \mathbf{x} d \xi+\int_{\mathcal{T}_{h}^{x}}\left|\varepsilon_{h}^{E}\right|^{2}+\left|\varepsilon_{h}^{B}\right|^{2} d \mathbf{x}\right)
$$

Here $\Lambda$ depends on $(f, \mathbf{E}, \mathbf{B})$ in their Sobolev norms $\left.\left\|\left.\partial_{t} f\right|_{k+1, \Omega},\right\| f\right|_{k+1, \Omega},|f|_{1, \infty, \Omega},\left\|\left.\mathbf{E}\right|_{1, \infty, \Omega_{x}},\right\| \mathbf{B} \|_{1, \infty, \Omega_{x}}$, $\|\mathbf{E}\|_{k+1, \Omega_{x}},\|\mathbf{B}\|_{k+1, \Omega_{x}}$ at time $t$, and $\Theta$ depends on $\|f\|_{k+1, \Omega}$ and $|f|_{1, \infty, \Omega}$ at time t. Both $\Lambda$ and $\Theta$ depend on the polynomial degree $k$, mesh parameters $\sigma_{0}, \sigma_{x}$ and $\sigma_{\xi}$, and domain parameters $L_{x}$ and $L_{\xi}$. Now with a standard application of Gronwall's inequality, a triangle inequality, and the approximation results of (4.1), we conclude the error estimate (4.4).

REMARK 4.4. Theorem 4.3 shows that the proposed methods are $\left(k+\frac{1}{2}\right)$-th order accurate, which is standard for upwind DG methods applied to hyperbolic problems on general meshes. The assumption on the polynomial degree $k \geq 2$ is due to the lack of the $L^{\infty}$ error estimate for the DG solutions to the Maxwell solver and the use of an inverse inequality in handling the nonlinear coupling (see (4.5)-(4.7) in the proof of Theorem 4.3). If the computational domain in $\mathbf{x}$ is one- or two-dimensional ( $d_{x}=1$ or 2 ), then Theorem 4.3 holds for $k \geq 1$. 
If the upwind numerical flux for the Maxwell solver $2.10 \mathrm{~b}$ is replaced by either the central or alternating flux 2.9a)-2.9b), we will have the estimates for $\varepsilon_{h}^{E}$ and $\varepsilon_{h}^{B}$ in Lemma 4.5 instead, provided an additional assumption is made for the mesh when it is refined. That is, we need to assume there is a positive constant $\delta<1$ such that for any $K_{x} \in \mathcal{T}_{h}^{x}$,

$$
\delta \leq \frac{h_{K_{x}{ }^{\prime}}}{h_{K_{x}}} \leq \frac{1}{\delta}
$$

where $K_{x}{ }^{\prime}$ is any element in $\mathcal{T}_{h}^{x}$ satisfying $K_{x}{ }^{\prime} \cap K_{x} \neq \emptyset$.

LEMMA 4.5 (Estimate of $\varepsilon_{h}^{E}$ and $\varepsilon_{h}^{B}$ with the non-upwinding flux). Based on the semi-discrete DG discretization for the Maxwell equations of 2.10b), with either the central or alternating flux of (2.9a)(2.9b), we have

$$
\begin{aligned}
\frac{d}{d t} \int_{\mathcal{T}_{h}^{x}}\left(\left|\varepsilon_{h}^{E}\right|^{2}+\left|\varepsilon_{h}^{B}\right|^{2}\right) d \mathbf{x} \lesssim & \left(\left\|\varepsilon_{h}\right\|_{0, \Omega}+h^{k+1}\|f\|_{k+1, \Omega}\right)\left\|\varepsilon_{h}^{E}\right\|_{0, \Omega_{x}} \\
& +c(\delta) h_{x}^{k}\left(\|\mathbf{E}\|_{k+1, \Omega_{x}}+\|\mathbf{B}\|_{k+1, \Omega_{x}}\right)\left(\int_{\mathcal{T}_{h}^{x}}\left(\left|\varepsilon_{h}^{E}\right|^{2}+\left|\varepsilon_{h}^{B}\right|^{2}\right) d \mathbf{x}\right)^{1 / 2}
\end{aligned}
$$

The proof of this Lemma is given in Subsection 4.3. With Lemma 4.5 and a proof similar to that of Theorem 4.3 the following error estimates can be established, but the proof is omitted.

THEOREM 4.6 (Error estimate 2). For $k \geq 2$, the semi-discrete DG method of (2.10a)-(2.10b) for Vlasov-Maxwell equations with the upwind numerical flux (2.8a)-(2.8b) for the Vlasov solver and either the central or alternating fluxes of 2.9a-2.9b for the Maxwell solver, has the following error estimate:

$$
\left\|\left(f-f_{h}\right)(t)\right\|_{0, \Omega}^{2}+\left\|\left(\mathbf{E}-\mathbf{E}_{h}\right)(t)\right\|_{0, \Omega_{x}}^{2}+\left\|\left(\mathbf{B}-\mathbf{B}_{h}\right)(t)\right\|_{0, \Omega_{x}}^{2} \leq C h^{2 k}, \quad \forall t \in[0, T] .
$$

Besides the dependence as in Theorem 4.3, the constant $C$ also depends on $\delta$ of (4.8).

Theorem 4.6 indicates that with either the central or alternating numerical flux for the Maxwell solver, the proposed method will be $k$-th order accurate. Also, one can see easily that the accuracy can be improved to $\left(k+\frac{1}{2}\right)$-th order as in Theorem 4.3 if the discrete space for Maxwell solver is one degree higher than that for the Vlasov equation, namely, $r=k+1$. This improvement will require higher regularity for the exact solution $\mathbf{E}$ and $\mathbf{B}$.

In 2], optimal error estimates were established for some DG methods solving the multi-dimensional Vlasov-Poisson problem on Cartesian meshes with tensor-structure discrete space, defined in (2.3), and $k \geq 1$. Some of the techniques in [2] are used in our analysis. In the present work, we focus on the P-type space $\mathcal{G}_{h}^{k}$ in 2.1a in the numerical section, as it renders better cost efficiency and can be used on more general meshes. Our analysis is established only for $k \geq 2$ due to the lack of the $L^{\infty}$ error estimate of the DG solver for the Maxwell part which is of hyperbolic nature, as pointed out in Remark 4.4

In the next three subsections, we will provide the proofs of Lemmas 4.1, 4.2 and 4.5 .

4.1. Proof of Lemma 4.1. Since the proposed method is consistent, the error equation is related to the Vlasov solver,

$$
a_{h}\left(f, \mathbf{E}, \mathbf{B} ; g_{h}\right)-a_{h}\left(f_{h}, \mathbf{E}_{h}, \mathbf{B}_{h} ; g_{h}\right)=0, \quad \forall g_{h} \in \mathcal{G}_{h}^{k}
$$

Note, $\varepsilon_{h} \in \mathcal{G}_{h}^{k}$; by taking $g_{h}=\varepsilon_{h}$ in 4.11, one has

$$
a_{h}\left(\varepsilon_{h}, \mathbf{E}_{h}, \mathbf{B}_{h} ; \varepsilon_{h}\right)=a_{h}\left(\Pi^{k} f, \mathbf{E}_{h}, \mathbf{B}_{h} ; \varepsilon_{h}\right)-a_{h}\left(f, \mathbf{E}, \mathbf{B} ; \varepsilon_{h}\right)
$$

Following the same lines as in the proof of Lemma 3.6, we get

$$
\begin{aligned}
a_{h}\left(\varepsilon_{h}, \mathbf{E}_{h}, \mathbf{B}_{h} ; \varepsilon_{h}\right)= & \frac{1}{2} \frac{d}{d t}\left(\int_{\mathcal{T}_{h}}\left|\varepsilon_{h}\right|^{2} d \mathbf{x} d \xi\right)+\frac{1}{2} \int_{\mathcal{T}_{h}^{\xi}} \int_{\mathcal{E}_{x}}\left|\xi \cdot \mathbf{n}_{x}\right|\left|\left[\varepsilon_{h}\right]_{x}\right|^{2} d s_{x} d \xi \\
& +\frac{1}{2} \int_{\mathcal{T}_{h}^{x}} \int_{\mathcal{E}_{\xi}}\left|\left(\mathbf{E}_{h}+\xi \times \mathbf{B}_{h}\right) \cdot \mathbf{n}_{\xi}\right|\left|\left[\varepsilon_{h}\right]_{\xi}\right|^{2} d s_{\xi} d \mathbf{x} .
\end{aligned}
$$


Next we will estimate the remaining terms in 4.12 . Note

$$
a_{h}\left(\Pi^{k} f, \mathbf{E}_{h}, \mathbf{B}_{h} ; \varepsilon_{h}\right)-a_{h}\left(f, \mathbf{E}, \mathbf{B} ; \varepsilon_{h}\right)=T_{1}+T_{2},
$$

where

$$
\begin{aligned}
& T_{1}=a_{h, 1}\left(\Pi^{k} f ; \varepsilon_{h}\right)-a_{h, 1}\left(f ; \varepsilon_{h}\right)=a_{h, 1}\left(\zeta_{h} ; \varepsilon_{h}\right) \\
& T_{2}=a_{h, 2}\left(\Pi^{k} f, \mathbf{E}_{h}, \mathbf{B}_{h} ; \varepsilon_{h}\right)-a_{h, 2}\left(f, \mathbf{E}, \mathbf{B} ; \varepsilon_{h}\right) .
\end{aligned}
$$

Step 1: estimate of $T_{1}$. We start with

$$
T_{1}=\int_{\mathcal{T}_{h}}\left(\partial_{t} \zeta_{h}\right) \varepsilon_{h} d \mathbf{x} d \xi-\int_{\mathcal{T}_{h}} \zeta_{h} \xi \cdot \nabla_{\mathbf{x}} \varepsilon_{h} d \mathbf{x} d \xi+\int_{\mathcal{T}_{h}^{\xi}} \int_{\mathcal{E}_{x}} \widetilde{\zeta_{h} \xi} \cdot\left[\varepsilon_{h}\right]_{x} d s_{x} d \xi=T_{11}+T_{12}+T_{13}
$$

It is easy to verify that $\partial_{t} \Pi^{k}=\Pi^{k} \partial_{t}$, and therefore $\partial_{t} \zeta_{h}=\Pi^{k}\left(\partial_{t} f\right)-\left(\partial_{t} f\right)$. With the approximation result of Lemma 2.1, we have

$$
\left|T_{11}\right|=\left|\int_{\mathcal{T}_{h}}\left(\partial_{t} \zeta_{h}\right) \varepsilon_{h} d \mathbf{x} d \xi\right| \leq\left\|\partial_{t} \zeta_{h}\right\|_{0, \Omega}\left\|\varepsilon_{h}\right\|_{0, \Omega} \lesssim h^{k+1}|| \partial_{t} f\left\|_{k+1, \Omega}\right\| \varepsilon_{h} \|_{0, \Omega}
$$

Next, let $\xi_{0}$ be the $L^{2}$ projection of the function $\xi$ onto the piecewise constant space with respect to $\mathcal{T}_{h}^{\xi}$, then

$$
T_{12}=-\int_{\mathcal{T}_{h}} \zeta_{h}\left(\xi-\xi_{0}\right) \cdot \nabla_{\mathbf{x}} \varepsilon_{h} d \mathbf{x} d \xi-\int_{\mathcal{T}_{h}} \zeta_{h} \xi_{0} \cdot \nabla_{\mathbf{x}} \varepsilon_{h} d \mathbf{x} d \xi
$$

Since $\xi_{0} \cdot \nabla_{\mathbf{x}} \varepsilon_{h} \in \mathcal{G}_{h}^{k}$ and $\zeta_{h}=\Pi^{k} f-f$ with $\Pi^{k}$ being the $L^{2}$ projection onto $\mathcal{G}_{h}^{k}$, the second term in 4.15 vanishes. Hence

$$
\begin{aligned}
\left|T_{12}\right| & \leq \int_{\mathcal{T}_{h}}\left|\zeta_{h}\left(\xi-\xi_{0}\right) \cdot \nabla_{\mathbf{x}} \varepsilon_{h}\right| d \mathbf{x} d \xi \\
& \leq\left\|\xi-\xi_{0}\right\|_{0, \infty, \Omega_{\xi}} \sum_{K_{x} \times K_{\xi}=K \in \mathcal{T}_{h}}\left(h_{K_{x}}^{-1}\left\|\zeta_{h}\right\|_{0, K}\right)\left(h_{K_{x}}\left\|\nabla_{\mathbf{x}} \varepsilon_{h}\right\|_{0, K}\right) \\
& \lesssim\left\|\xi-\xi_{0}\right\|_{0, \infty, \Omega_{\xi}} \sum_{K_{x} \times K_{\xi}=K \in \mathcal{T}_{h}} h_{K}^{k+1} h_{K_{x}}^{-1}\|f\|_{k+1, K}\left\|\varepsilon_{h}\right\|_{0, K} \\
& \lesssim h_{\xi}\|\xi\|_{1, \infty, \Omega_{\xi}} h^{k}\|f\|_{k+1, \Omega}\left\|\varepsilon_{h}\right\|_{0, \Omega} \\
& \lesssim h^{k+1}\|f\|_{k+1, \Omega}\left\|\varepsilon_{h}\right\|_{0, \Omega} .
\end{aligned}
$$

The third inequality above uses the approximating result of Lemma 2.1 and the inverse inequality of Lemma 2.2 The fourth inequality uses an approximation result similar to the last one of Lemma 2.1 and $\frac{h_{\xi}}{h_{x, \min }}$ being uniformly bounded by $\sigma_{0}$ when the mesh is refined.

Next,

$$
\begin{aligned}
T_{13} & =\int_{\mathcal{T}_{h}^{\xi}} \int_{\mathcal{E}_{x}}\left(\left\{\zeta_{h}\right\}_{x} \xi+\frac{\left|\xi \cdot \mathbf{n}_{x}\right|}{2}\left[\zeta_{h}\right]_{x}\right) \cdot\left[\varepsilon_{h}\right]_{x} d s_{x} d \xi \\
& =\int_{\mathcal{T}_{h}^{\xi}} \int_{\mathcal{E}_{x}}\left(\left\{\zeta_{h}\right\}_{x}\left(\xi \cdot \hat{\mathbf{n}}_{x}\right) \hat{\mathbf{n}}_{x}+\frac{\left|\xi \cdot \mathbf{n}_{x}\right|}{2}\left[\zeta_{h}\right]_{x}\right) \cdot\left[\varepsilon_{h}\right]_{x} d s_{x} d \xi,
\end{aligned}
$$


where $\hat{\mathbf{n}}_{x}$ is the unit normal vector of an edge in $\mathcal{E}_{x}$ with either orientation, that is $\hat{\mathbf{n}}_{x}=\mathbf{n}_{x}$, or $-\mathbf{n}_{x}$. Then,

$$
\begin{aligned}
\left|T_{13}\right| & \leq \int_{\mathcal{T}_{h}^{\xi}} \int_{\mathcal{E}_{x}}\left(\left|\xi \cdot \mathbf{n}_{x}\right|\left(\left|\left\{\zeta_{h}\right\}_{x}\right|+\frac{\left|\left[\zeta_{h}\right]_{x}\right|}{2}\right)\right) \cdot\left|\left[\varepsilon_{h}\right]_{x}\right| d s_{x} d \xi \\
& \leq\left(\int_{\mathcal{T}_{h}^{\xi}} \int_{\mathcal{E}_{x}} 2\left(\left|\left\{\zeta_{h}\right\}_{x}\right|^{2}+\left(\frac{\left|\left[\zeta_{h}\right]_{x}\right|}{2}\right)^{2}\right)\left|\xi \cdot \mathbf{n}_{x}\right| d s_{x} d \xi\right)^{1 / 2}\left(\int_{\mathcal{T}_{h}^{\xi}} \int_{\mathcal{E}_{x}}\left|\xi \cdot \mathbf{n}_{x}\right|\left|\left[\varepsilon_{h}\right]_{x}\right|^{2} d s_{x} d \xi\right)^{1 / 2} \\
& =\left(\int_{\mathcal{T}_{h}^{\xi}} \int_{\mathcal{E}_{x}} 2\left|\xi \cdot \mathbf{n}_{x}\right|\left|\left\{\zeta_{h}^{2}\right\}_{x}\right| d s_{x} d \xi\right)^{1 / 2}\left(\int_{\mathcal{T}_{h}^{\xi}} \int_{\mathcal{E}_{x}}\left|\xi \cdot \mathbf{n}_{x}\right|\left|\left[\varepsilon_{h}\right]_{x}\right|^{2} d s_{x} d \xi\right)^{1 / 2} \\
& \lesssim\|\xi\|_{0, \infty, \Omega_{\xi}}^{1 / 2} \| \zeta_{h}||_{0, \mathcal{T}_{h}^{\xi} \times \mathcal{E}_{x}}\left(\int_{\mathcal{T}_{h}^{\xi}} \int_{\mathcal{E}_{x}}\left|\xi \cdot \mathbf{n}_{x}\right|\left|\left[\varepsilon_{h}\right]_{x}\right|^{2} d s_{x} d \xi\right)^{1 / 2} \\
& \lesssim h^{k+\frac{1}{2}}\|f\|_{k+1, \Omega}\left(\int_{\mathcal{T}_{h}^{\xi}} \int_{\mathcal{E}_{x}}\left|\xi \cdot \mathbf{n}_{x}\right|\left|\left[\varepsilon_{h}\right]_{x}\right|^{2} d s_{x} d \xi\right)^{1 / 2}
\end{aligned}
$$

The approximation results of Lemma 2.1 are used for the last inequality.

Step 2: estimate of $T_{2}$. Note,

$$
\begin{aligned}
T_{2} & =a_{h, 2}\left(\Pi^{k} f, \mathbf{E}_{h}, \mathbf{B}_{h} ; \varepsilon_{h}\right)-a_{h, 2}\left(f, \mathbf{E}, \mathbf{B} ; \varepsilon_{h}\right) \\
& =a_{h, 2}\left(\zeta_{h}, \mathbf{E}_{h}, \mathbf{B}_{h} ; \varepsilon_{h}\right)+a_{h, 2}\left(f, \mathbf{E}_{h}, \mathbf{B}_{h} ; \varepsilon_{h}\right)-a_{h, 2}\left(f, \mathbf{E}, \mathbf{B} ; \varepsilon_{h}\right)=T_{21}+T_{22}+T_{23},
\end{aligned}
$$

with

$$
\begin{aligned}
& T_{21}=-\int_{\mathcal{T}_{h}} \zeta_{h}\left(\mathbf{E}_{h}+\xi \times \mathbf{B}_{h}\right) \cdot \nabla_{\xi} \varepsilon_{h} d \mathbf{x} d \xi, \quad T_{22}=\int_{\mathcal{T}_{h}^{x}} \int_{\mathcal{E}_{\xi}} \zeta_{h}\left(\widetilde{\mathbf{E}_{h}} \widetilde{+\xi} \times \mathbf{B}_{h}\right) \cdot\left[\varepsilon_{h}\right]_{\xi} d s_{\xi} d x, \\
& T_{23}=a_{h, 2}\left(f, \mathbf{E}_{h}, \mathbf{B}_{h} ; \varepsilon_{h}\right)-a_{h, 2}\left(f, \mathbf{E}, \mathbf{B} ; \varepsilon_{h}\right) .
\end{aligned}
$$

For $T_{21}$, we proceed as for the estimate of $T_{12}$. Let $\mathbf{E}_{0}=\Pi_{x}^{0} \mathbf{E}, \mathbf{B}_{0}=\boldsymbol{\Pi}_{x}^{0} \mathbf{B}$ be the $L^{2}$ projection of $\mathbf{E}, \mathbf{B}$, respectively, onto the piecewise constant vector space with respect to $\mathcal{T}_{h}^{x}$, then

$$
\begin{aligned}
\int_{\mathcal{T}_{h}} \zeta_{h}\left(\mathbf{E}_{h}+\xi \times \mathbf{B}_{h}\right) \cdot \nabla_{\xi} \varepsilon_{h} d \mathbf{x} d \xi= & \int_{\mathcal{T}_{h}} \zeta_{h}\left(\mathbf{E}_{h}-\mathbf{E}_{0}+\xi \times\left(\mathbf{B}_{h}-\mathbf{B}_{0}\right)\right) \cdot \nabla_{\xi} \varepsilon_{h} d \mathbf{x} d \xi \\
& +\int_{\mathcal{T}_{h}} \zeta_{h}\left(\mathbf{E}_{0}+\xi \times \mathbf{B}_{0}\right) \cdot \nabla_{\xi} \varepsilon_{h} d \mathbf{x} d \xi
\end{aligned}
$$

and the second term above vanishes due to $\left(\mathbf{E}_{0}+\xi \times \mathbf{B}_{0}\right) \cdot \nabla_{\xi} \varepsilon_{h} \in \mathcal{G}_{h}^{k}$, and therefore

$$
\begin{aligned}
& \left|\int_{\mathcal{T}_{h}} \zeta_{h}\left(\mathbf{E}_{h}+\xi \times \mathbf{B}_{h}\right) \cdot \nabla_{\xi} \varepsilon_{h} d \mathbf{x} d \xi\right| \leq \int_{\mathcal{T}_{h}}\left|\zeta_{h}\left(\mathbf{E}_{h}-\mathbf{E}_{0}+\xi \times\left(\mathbf{B}_{h}-\mathbf{B}_{0}\right)\right) \cdot \nabla_{\xi} \varepsilon_{h}\right| d \mathbf{x} d \xi, \\
& \leq\left(\left\|\mathbf{E}_{h}-\mathbf{E}_{0}+\xi \times\left(\mathbf{B}_{h}-\mathbf{B}_{0}\right)\right\|_{0, \infty, \Omega}\right) \sum_{K_{x} \times K_{\xi}=K \in \mathcal{T}_{h}}\left(h_{K_{\xi}}^{-1}\left\|\zeta_{h}\right\|_{0, K}\right)\left(h_{K_{\xi}}\left\|\nabla_{\xi} \varepsilon_{h}\right\|_{0, K}\right), \\
& \lesssim\left(\left\|\mathbf{E}_{h}-\mathbf{E}_{0}\right\|_{0, \infty, \Omega_{x}}+\left\|\left(\mathbf{B}_{h}-\mathbf{B}_{0}\right)\right\|_{0, \infty, \Omega_{x}}\right) \sum_{K_{x} \times K_{\xi}=K \in \mathcal{T}_{h}} h_{K}^{k+1} h_{K_{\xi}}^{-1}\|f\|_{k+1, K}\left\|\varepsilon_{h}\right\|_{0, K}, \\
& \lesssim h^{k}\|f\|_{k+1, \Omega}\left(\left\|\varepsilon_{h}^{E}\right\|_{0, \infty, \Omega_{x}}+\left\|\varepsilon_{h}^{B}\right\|_{0, \infty, \Omega_{x}}+\left\|\mathbf{\Pi}_{x}^{k} \mathbf{E}-\mathbf{E}_{0}\right\|_{0, \infty, \Omega_{x}}+\left\|\mathbf{\Pi}_{x}^{k} \mathbf{B}-\mathbf{B}_{0}\right\|_{0, \infty, \Omega_{x}}\right)\left\|\varepsilon_{h}\right\|_{0, \Omega} .
\end{aligned}
$$

Note that $\boldsymbol{\Pi}_{x}^{k} \mathbf{E}-\mathbf{E}_{0}=\boldsymbol{\Pi}_{x}^{k}\left(\mathbf{E}-\mathbf{E}_{0}\right)$, and $\boldsymbol{\Pi}_{x}^{k}$ is bounded in any $L^{p}$-norm $(1 \leq p \leq \infty)$,20, 2], then

$$
\left\|\mathbf{\Pi}_{x}^{k} \mathbf{E}-\mathbf{E}_{0}\right\|_{0, \infty, \Omega_{x}} \lesssim\left\|\mathbf{E}-\mathbf{E}_{0}\right\|_{0, \infty, \Omega_{x}} \lesssim h_{x}\|\mathbf{E}\|_{1, \infty, \Omega_{x}}
$$


and similarly $\left\|\boldsymbol{\Pi}_{x}^{k} \mathbf{B}-\mathbf{B}_{0}\right\|_{0, \infty, \Omega_{x}} \lesssim h_{x}\|\mathbf{B}\|_{1, \infty, \Omega_{x}}$. Hence

$$
\begin{aligned}
&\left|\int_{\mathcal{T}_{h}} \zeta_{h}\left(\mathbf{E}_{h}+\xi \times \mathbf{B}_{h}\right) \cdot \nabla_{\xi} \varepsilon_{h} d \mathbf{x} d \xi\right| \\
& \lesssim h^{k}|| f\left\|_{k+1, \Omega}\left(\left\|\varepsilon_{h}^{E}\right\|_{0, \infty, \Omega_{x}}+\left\|\varepsilon_{h}^{B}\right\|_{0, \infty, \Omega_{x}}+h_{x}\left(\|\mathbf{E}\|_{1, \infty, \Omega_{x}}+\|\mathbf{B}\|_{1, \infty, \Omega_{x}}\right)\right)\right\| \varepsilon_{h} \|_{0, \Omega} .
\end{aligned}
$$

For $T_{22}$, we follow the estimate of $T_{13}$. Note that $\mathbf{E}_{h}$ and $\mathbf{B}_{h}$ only depends on $\mathbf{x}$, and $\xi$ is continuous,

$$
\begin{aligned}
& \left|\int_{\mathcal{T}_{h}^{x}} \int_{\mathcal{E}_{\xi}} \zeta_{h}\left(\mathbf{E}_{h}+\xi \times \mathbf{B}_{h}\right) \cdot\left[\varepsilon_{h}\right]_{\xi} d s_{\xi} d x\right| \\
& =\left|\int_{\mathcal{T}_{h}^{x}} \int_{\mathcal{E}_{\xi}}\left(\left\{\zeta_{h}\left(\mathbf{E}_{h}+\xi \times \mathbf{B}_{h}\right)\right\}_{\xi}+\frac{\left|\left(\mathbf{E}_{h}+\xi \times \mathbf{B}_{h}\right) \cdot \mathbf{n}_{\xi}\right|}{2}\left[\zeta_{h}\right]_{\xi}\right) \cdot\left[\varepsilon_{h}\right]_{\xi} d s_{\xi} d x\right|, \\
& =\left|\int_{\mathcal{T}_{h}^{x}} \int_{\mathcal{E}_{\xi}}\left(\left\{\zeta_{h}\right\}_{\xi}\left(\left(\mathbf{E}_{h}+\xi \times \mathbf{B}_{h}\right) \cdot \hat{\mathbf{n}}_{\xi}\right) \hat{\mathbf{n}}_{\xi}+\frac{\left|\left(\mathbf{E}_{h}+\xi \times \mathbf{B}_{h}\right) \cdot \mathbf{n}_{\xi}\right|}{2}\left[\zeta_{h}\right]_{\xi}\right) \cdot\left[\varepsilon_{h}\right]_{\xi} d s_{\xi} d x\right|, \quad \hat{\mathbf{n}}_{\xi}=\mathbf{n}_{\xi} \text { or }-\mathbf{n}_{\xi} \\
& \leq \int_{\mathcal{T}_{h}^{x}} \int_{\mathcal{E}_{\xi}}\left(\left|\left(\mathbf{E}_{h}+\xi \times \mathbf{B}_{h}\right) \cdot \mathbf{n}_{\xi}\right|\left(\left|\left\{\zeta_{h}\right\}_{\xi}\right|+\left|\frac{\left[\zeta_{h}\right]_{\xi}}{2}\right|\right)\right)\left|\left[\varepsilon_{h}\right]_{\xi}\right| d s_{\xi} d x, \\
& \leq\left(\int_{\mathcal{T}_{h}^{x}} \int_{\mathcal{E}_{\xi}} 2\left|\left(\mathbf{E}_{h}+\xi \times \mathbf{B}_{h}\right) \cdot \mathbf{n}_{\xi}\right|\left|\left\{\zeta_{h}^{2}\right\}\right| d s_{\xi} d x\right)^{1 / 2}\left(\int_{\mathcal{T}_{h}^{x}} \int_{\mathcal{E}_{\xi}}\left|\left(\mathbf{E}_{h}+\xi \times \mathbf{B}_{h}\right) \cdot \mathbf{n}_{\xi}\right|\left|\left[\varepsilon_{h}\right]\right|^{2} d s_{\xi} d x\right)^{1 / 2} .
\end{aligned}
$$

In addition,

$$
\begin{aligned}
& \left(\int_{\mathcal{T}_{h}^{x}} \int_{\mathcal{E}_{\xi}} 2\left|\left(\mathbf{E}_{h}+\xi \times \mathbf{B}_{h}\right) \cdot \mathbf{n}_{\xi} \|\left\{\zeta_{h}^{2}\right\}\right| d s_{\xi} d x\right)^{1 / 2} \\
& \lesssim\left\|\mathbf{E}_{h}+\xi \times \mathbf{B}_{h}\right\|_{0, \infty, \Omega}^{1 / 2}\left\|\zeta_{h}\right\|_{0, \mathcal{T}_{h}^{x} \times \mathcal{E}_{\xi}} \\
& \lesssim\left\|\zeta_{h}\right\|_{0, \mathcal{T}_{h}^{x} \times \mathcal{E}_{\xi}}\left(\left\|\mathbf{E}_{h}\right\|_{0, \infty, \Omega_{x}}^{1 / 2}+\left\|\mathbf{B}_{h}\right\|_{0, \infty, \Omega_{x}}^{1 / 2}\right) \\
& \lesssim h^{k+\frac{1}{2}}\|f\|_{k+1, \Omega}\left(\left\|\varepsilon_{h}^{E}\right\|_{0, \infty, \Omega_{x}}^{1 / 2}+\left\|\varepsilon_{h}^{B}\right\|_{0, \infty, \Omega_{x}}^{1 / 2}+\|\mathbf{E}\|_{0, \infty, \Omega_{x}}^{1 / 2}+\|\mathbf{B}\|_{0, \infty, \Omega_{x}}^{1 / 2}\right),
\end{aligned}
$$

and therefore

$$
\begin{gathered}
T_{22} \lesssim h^{k+\frac{1}{2}}\|f\|_{k+1, \Omega}\left(\left\|\varepsilon_{h}^{E}\right\|_{0, \infty, \Omega_{x}}^{1 / 2}+\left\|\varepsilon_{h}^{B}\right\|_{0, \infty, \Omega_{x}}^{1 / 2}+\|\mathbf{E}\|_{0, \infty, \Omega_{x}}^{1 / 2}+\|\mathbf{B}\|_{0, \infty, \Omega_{x}}^{1 / 2}\right) \\
\left(\int_{\mathcal{T}_{h}^{x}} \int_{\mathcal{E}_{\xi}}\left|\left(\mathbf{E}_{h}+\xi \times \mathbf{B}_{h}\right) \cdot \mathbf{n}_{\xi} \|\left[\varepsilon_{h}\right]\right|^{2} d s_{\xi} d x\right)^{1 / 2} .
\end{gathered}
$$

Finally, we estimate $T_{23}$. Since $f$ is continuous in $\xi$, and $\nabla_{\xi} \cdot\left(\mathbf{E}_{h}-\mathbf{E}+\xi \times\left(\mathbf{B}_{h}-\mathbf{B}\right)\right)=0$,

$$
\begin{aligned}
T_{23} & =a_{h, 2}\left(f, \mathbf{E}_{h}, \mathbf{B}_{h} ; \varepsilon_{h}\right)-a_{h, 2}\left(f, \mathbf{E}, \mathbf{B} ; \varepsilon_{h}\right) \\
& =-\int_{\mathcal{T}_{h}} f\left(\mathbf{E}_{h}-\mathbf{E}+\xi \times\left(\mathbf{B}_{h}-\mathbf{B}\right)\right) \cdot \nabla_{\xi} \varepsilon_{h} d \mathbf{x} d \xi+\int_{\mathcal{T}_{h}^{x}} \int_{\mathcal{E}_{\xi}} f\left(\mathbf{E}_{h}-\mathbf{E}+\xi \times\left(\mathbf{B}_{h}-\mathbf{B}\right)\right) \cdot\left[\varepsilon_{h}\right]_{\xi} d s_{\xi} d x, \\
& =\int_{\mathcal{T}_{h}} \nabla_{\xi} f \cdot\left(\mathbf{E}_{h}-\mathbf{E}+\xi \times\left(\mathbf{B}_{h}-\mathbf{B}\right)\right) \varepsilon_{h} d \mathbf{x} d \xi ;
\end{aligned}
$$

therefore,

$$
\begin{aligned}
\left|T_{23}\right| & \leq\left\|\mathbf{E}_{h}-\mathbf{E}+\xi \times\left(\mathbf{B}_{h}-\mathbf{B}\right)\right\|_{0, \Omega}|f|_{1, \infty, \Omega}\left\|\varepsilon_{h}\right\|_{0, \Omega}, \\
& \lesssim\left(\left\|\mathbf{E}_{h}-\mathbf{E}\right\|_{0, \Omega_{x}}+\left\|\left(\mathbf{B}_{h}-\mathbf{B}\right)\right\|_{0, \Omega_{x}}\right)|f|_{1, \infty, \Omega}\left\|\varepsilon_{h}\right\|_{0, \Omega}, \\
& \lesssim\left(\left\|\varepsilon_{h}^{E}\right\|_{0, \Omega_{x}}+\left\|\varepsilon_{h}^{B}\right\|_{0, \Omega_{x}}+\left\|\boldsymbol{\zeta}_{h}^{E}\right\|_{0, \Omega_{x}}+\left\|\boldsymbol{\zeta}_{h}^{B}\right\|_{0, \Omega_{x}}\right)|f|_{1, \infty, \Omega}\left\|\varepsilon_{h}\right\|_{0, \Omega}, \\
& \lesssim\left(\left\|\varepsilon_{h}^{E}\right\|_{0, \Omega_{x}}+\left\|\varepsilon_{h}^{B}\right\|_{0, \Omega_{x}}+h_{x}^{k+1}\left(\left\|\left.\mathbf{E}\right|_{k+1, \Omega_{x}}+\right\| \mathbf{B} \|_{k+1, \Omega_{x}}\right)\right)|f|_{1, \infty, \Omega}\left\|\varepsilon_{h}\right\|_{0, \Omega} .
\end{aligned}
$$

Now we combine the estimates of (4.14) and 4.16)- 4.20 , and get the result of Lemma 4.1 . 
4.2. Proof of Lemma 4.2. Since the proposed method is consistent, the error equation is related to the Maxwell solver,

$$
b_{h}\left(\mathbf{E}-\mathbf{E}_{h}, \mathbf{B}-\mathbf{B}_{h} ; \mathbf{U}, \mathbf{V}\right)=l_{h}\left(\mathbf{J}-\mathbf{J}_{h}, \mathbf{U}\right), \quad \forall \mathbf{U}, \mathbf{V} \in \mathcal{U}_{h}^{k} .
$$

Taking the test functions in 4.21 to be $\mathbf{U}=\varepsilon_{h}^{E}$ and $\mathbf{V}=\varepsilon_{h}^{B}$ gives

$$
b_{h}\left(\varepsilon_{h}^{E}, \varepsilon_{h}^{B} ; \varepsilon_{h}^{E}, \varepsilon_{h}^{B}\right)=b_{h}\left(\boldsymbol{\zeta}_{h}^{E}, \boldsymbol{\zeta}_{h}^{B} ; \varepsilon_{h}^{E}, \varepsilon_{h}^{B}\right)+l_{h}\left(\mathbf{J}-\mathbf{J}_{h}, \varepsilon_{h}^{E}\right) .
$$

Following the same lines of Step 2 in the proof of Lemma 3.2 .

$$
b_{h}\left(\varepsilon_{h}^{E}, \varepsilon_{h}^{B} ; \varepsilon_{h}^{E}, \varepsilon_{h}^{B}\right)=\frac{1}{2} \frac{d}{d t} \int_{\mathcal{T}_{h}^{x}}\left(\left|\varepsilon_{h}^{E}\right|^{2}+\left|\varepsilon_{h}^{B}\right|^{2}\right) d \mathbf{x}+\frac{1}{2} \int_{\mathcal{E}_{x}}\left(\left|\left[\varepsilon_{h}^{E}\right]_{\tau}\right|^{2}+\left|\left[\varepsilon_{h}^{B}\right]_{\tau}\right|^{2}\right) d s_{x}
$$

It remains to estimate the two terms on the right side of 4.22 ,

$$
\begin{aligned}
& b_{h}\left(\boldsymbol{\zeta}_{h}^{E}, \boldsymbol{\zeta}_{h}^{B} ; \varepsilon_{h}^{E}, \boldsymbol{\varepsilon}_{h}^{B}\right) \\
= & \int_{\mathcal{T}_{h}^{x}} \partial_{t} \boldsymbol{\zeta}_{h}^{E} \cdot \varepsilon_{h}^{E} d \mathbf{x}-\int_{\mathcal{T}_{h}^{x}} \boldsymbol{\zeta}_{h}^{B} \cdot \nabla \times \varepsilon_{h}^{E} d \mathbf{x}-\int_{\mathcal{E}_{x}} \widetilde{\boldsymbol{\zeta}_{h}^{B}} \cdot\left[\varepsilon_{h}^{E}\right]_{\tau} d s_{x} \\
& +\int_{\mathcal{T}_{h}^{x}} \partial_{t} \boldsymbol{\zeta}_{h}^{B} \cdot \varepsilon_{h}^{B} d \mathbf{x}+\int_{\mathcal{T}_{h}^{x}} \boldsymbol{\zeta}_{h}^{E} \cdot \nabla \times \varepsilon_{h}^{B} d \mathbf{x}+\int_{\mathcal{E}_{x}} \widetilde{\boldsymbol{\zeta}_{h}^{E}} \cdot\left[\varepsilon_{h}^{B}\right]_{\tau} d s_{x}, \\
= & -\int_{\mathcal{E}_{x}} \widetilde{\boldsymbol{\zeta}_{h}^{B}} \cdot\left[\varepsilon_{h}^{E}\right]_{\tau} d s_{x}+\int_{\mathcal{E}_{x}} \widetilde{\boldsymbol{\zeta}_{h}^{E}} \cdot\left[\varepsilon_{h}^{B}\right]_{\tau} d s_{x} \\
\leq & \left(\int_{\mathcal{E}_{x}}\left|\widetilde{\boldsymbol{\zeta}_{h}^{B}}\right|^{2}+\left|\widetilde{\boldsymbol{\zeta}_{h}^{E}}\right|^{2} d s_{x}\right)^{1 / 2}\left(\int_{\mathcal{E}_{x}}\left|\left[\varepsilon_{h}^{E}\right]_{\tau}\right|^{2}+\left|\left[\varepsilon_{h}^{B}\right]_{\tau}\right|^{2} d s_{x}\right)^{1 / 2} \\
\lesssim & \sum_{K_{x} \in \mathcal{T}_{h}^{x}}\left(\left\|\boldsymbol{\zeta}_{h}^{E}\right\|_{0, \partial K_{x}}+\left\|\boldsymbol{\zeta}_{h}^{B}\right\|_{0, \partial K_{x}}\right)\left(\int_{\mathcal{E}_{x}}\left|\left[\varepsilon_{h}^{E}\right]_{\tau}\right|^{2}+\left|\left[\varepsilon_{h}^{B}\right]_{\tau}\right|^{2} d s_{x}\right)^{1 / 2} \\
\lesssim & h_{x}^{k+\frac{1}{2}}\left(\|\mathbf{E}\|_{k+1, \Omega_{x}}+\|\mathbf{B}\|_{k+1, \Omega_{x}}\right)\left(\int_{\mathcal{E}_{x}}\left|\left[\varepsilon_{h}^{E}\right]_{\tau}\right|^{2}+\left|\left[\varepsilon_{h}^{B}\right]_{\tau}\right|^{2} d s_{x}\right)^{1 / 2}
\end{aligned}
$$

All of the volume integrals of (4.24) vanish due to $\partial_{t} \Pi_{x}^{k}=\Pi_{x}^{k} \partial_{t}$ and $\varepsilon_{h}^{E}, \varepsilon_{h}^{B}, \nabla \times \varepsilon_{h}^{E}, \nabla \times \varepsilon_{h}^{B} \in \mathcal{U}_{h}^{k}$. And, for the last two inequalities, the definition of the numerical fluxes are used together with the approximation results of Lemma 2.1. Finally,

$$
\begin{aligned}
\left|l_{h}\left(\mathbf{J}-\mathbf{J}_{h} ; \varepsilon_{h}^{E}\right)\right| & =\left|\int_{\mathcal{T}_{h}^{x}}\left(\mathbf{J}-\mathbf{J}_{h}\right) \cdot \varepsilon_{h}^{E} d \mathbf{x}\right| \\
& \leq\left\|\mathbf{J}-\mathbf{J}_{h}\right\|_{0, \Omega_{x}}\left\|\varepsilon_{h}^{E}\right\|_{0, \Omega_{x}}=\left\|\int_{\mathcal{T}_{\xi}}\left(f-f_{h}\right) \xi d \xi\right\|_{0, \Omega_{x}}\left\|\varepsilon_{h}^{E}\right\|_{0, \Omega_{x}}, \\
& \leq\left\|f-f_{h}\right\|_{0, \Omega}\|\xi\|_{0, \Omega_{\xi}}\left\|\varepsilon_{h}^{E}\right\|_{0, \Omega_{x}}, \\
& \lesssim\left(\left\|\varepsilon_{h}\right\|_{0, \Omega}+\left\|\zeta_{h}\right\|_{0, \Omega}\right)\left\|\varepsilon_{h}^{E}\right\|_{0, \Omega_{x}} \lesssim\left(\left\|\varepsilon_{h}\right\|_{0, \Omega}+h^{k+1}\|f\|_{k+1, \Omega}\right)\left\|\varepsilon_{h}^{E}\right\|_{0, \Omega_{x}}
\end{aligned}
$$

Combining 4.23)-4.25, we conclude Lemma 4.2 .

4.3. Proof of Lemma 4.5. The proof proceeds in a manner similar to that of Lemma 4.2 of Subsection 4.2. Based on the error equation (4.21), related to the Maxwell solver with some specific test functions, we get 4.22 . With either the central or alternating flux of $2.9 \mathrm{a})-2.9 \mathrm{~b})$, we have

$$
b_{h}\left(\varepsilon_{h}^{E}, \varepsilon_{h}^{B} ; \varepsilon_{h}^{E}, \varepsilon_{h}^{B}\right)=\frac{1}{2} \frac{d}{d t} \int_{\mathcal{T}_{h}^{x}}\left(\left|\varepsilon_{h}^{E}\right|^{2}+\left|\varepsilon_{h}^{B}\right|^{2}\right) d \mathbf{x} .
$$


The same estimate as that of 4.25) can be obtained for the second term on the right of 4.22 . To estimate the first one,

$$
\begin{aligned}
& b_{h}\left(\boldsymbol{\zeta}_{h}^{E}, \boldsymbol{\zeta}_{h}^{B} ; \varepsilon_{h}^{E}, \boldsymbol{\varepsilon}_{h}^{B}\right) \\
= & \int_{\mathcal{T}_{h}^{x}} \partial_{t} \boldsymbol{\zeta}_{h}^{E} \cdot \varepsilon_{h}^{E} d \mathbf{x}-\int_{\mathcal{T}_{h}^{x}} \boldsymbol{\zeta}_{h}^{B} \cdot \nabla \times \varepsilon_{h}^{E} d \mathbf{x}-\int_{\mathcal{E}_{x}} \widetilde{\boldsymbol{\zeta}_{h}^{B}} \cdot\left[\varepsilon_{h}^{E}\right]_{\tau} d s_{x} \\
& +\int_{\mathcal{T}_{h}^{x}} \partial_{t} \boldsymbol{\zeta}_{h}^{B} \cdot \varepsilon_{h}^{B} d \mathbf{x}+\int_{\mathcal{T}_{h}^{x}} \boldsymbol{\zeta}_{h}^{E} \cdot \nabla \times \boldsymbol{\varepsilon}_{h}^{B} d \mathbf{x}+\int_{\mathcal{E}_{x}} \widetilde{\boldsymbol{\zeta}_{h}^{E}} \cdot\left[\varepsilon_{h}^{B}\right]_{\tau} d s_{x} \\
= & -\int_{\mathcal{E}_{x}} \widetilde{\boldsymbol{\zeta}_{h}^{B}} \cdot\left[\varepsilon_{h}^{E}\right]_{\tau} d s_{x}+\int_{\mathcal{E}_{x}} \widetilde{\boldsymbol{\zeta}_{h}^{E}} \cdot\left[\varepsilon_{h}^{B}\right]_{\tau} d s_{x} \\
\leq & \left(\sum_{e \in \mathcal{E}_{x}} \int_{e} h_{K_{x}}^{-1}\left(\left|\widetilde{\boldsymbol{\zeta}_{h}^{B}}\right|^{2}+\left|\widetilde{\boldsymbol{\zeta}_{h}^{E}}\right|^{2}\right) d s_{x}\right)^{1 / 2}\left(\sum_{e \in \mathcal{E}_{x}} \int_{e} h_{K_{x}}\left(\left|\left[\varepsilon_{h}^{E}\right]_{\tau}\right|^{2}+\left|\left[\varepsilon_{h}^{B}\right]_{\tau}\right|^{2}\right) d s_{x}\right)^{1 / 2}\left(\sum_{K_{x} \in \mathcal{T}_{h}^{x}} \int_{\partial K_{x}} h_{K_{x}}\left(\left|\varepsilon_{h}^{E}\right|^{2}+\left|\varepsilon_{h}^{B}\right|^{2}\right) d s_{x}\right)^{1 / 2} \\
\lesssim & c(\delta)\left(\sum_{K_{x} \in \mathcal{T}_{h}^{x}} \int_{\partial K_{x}} h_{K_{x}}^{-1}\left(\left|\boldsymbol{\zeta}_{h}^{B}\right|^{2}+\left|\boldsymbol{\zeta}_{h}^{E}\right|^{2}\right) d s_{x}\right)^{1 / 2}\left(\sum_{K_{x} \in \mathcal{T}_{h}^{x}}\left(\left\|\varepsilon_{h}^{E}\right\|_{0, K_{x}}^{2}+\left\|\varepsilon_{h}^{B}\right\|_{0, K_{x}}^{2}\right)\right)^{1 / 2} \\
\lesssim & c(\delta)\left(\sum_{K_{x} \in \mathcal{T}_{h}^{x}} h_{K_{x}}^{2 k}\left(\|\mathbf{E}\|_{k+1, K_{x}}^{2}+\|\mathbf{B}\|_{k+1, K_{x}}^{2}\right)\right)^{1 / 2} \\
\lesssim & c(\delta) h_{x}^{k}\left(\|\mathbf{E}\|_{k+1, \Omega_{x}}+\|\mathbf{B}\|_{k+1, \Omega_{x}}\right)\left(\int_{\mathcal{T}_{h}^{x}}\left(\left|\varepsilon_{h}^{E}\right|^{2}+\left|\varepsilon_{h}^{B}\right|^{2}\right) d \mathbf{x}\right)^{.}
\end{aligned}
$$

As before, all volume integrals of 4.26 vanish due to $\partial_{t} \Pi_{x}^{k}=\Pi_{x}^{k} \partial_{t}$ and $\varepsilon_{h}^{E}, \varepsilon_{h}^{B}, \nabla \times \varepsilon_{h}^{E}, \nabla \times \varepsilon_{h}^{B} \in \mathcal{U}_{h}^{k}$. In (4.27), $K_{x}$ is any element containing an edge $e$. To get 4.28), we use the definitions of the numerical fluxes, jumps, as well as the assumption (4.8) on the ratio of the neighboring mesh elements. Here $c(\delta)$ is a positive constant depending on $\delta$. We obtain 4.29 by applying an approximation result of Lemma 2.1 and an inverse inequality of Lemma 2.2 . From all the above, we conclude Lemma 4.5 .

5. Numerical results. In this section, we perform a detailed numerical study of the proposed scheme in the context of the streaming Weibel (SW) instability first analyzed in 48. The SW instability is closely related to the Weibel instability of [57, but derives its free energy from transverse counter-streaming as opposed to temperature anisotropy. The SW instability and its Weibel counterpart have been considered both analytically and numerically in several papers (e.g. 48, 9, 8, 7, 47,) - here we focus on comparison with the numerical results of Califano et al. in 9 .

We consider a reduced version of the Vlasov-Maxwell equations with one spatial variable, $x_{2}$, and two velocity variables, $\xi_{1}$ and $\xi_{2}$, The dependent variables under consideration are the distribution function $f\left(x_{2}, \xi_{1}, \xi_{2}, t\right)$, a 2D electric field $\mathbf{E}=\left(E_{1}\left(x_{2}, t\right), E_{2}\left(x_{2}, t\right), 0\right)$ and a $1 \mathrm{D}$ magnetic field $\mathbf{B}=\left(0,0, B_{3}\left(x_{2}, t\right)\right)$, and the reduced Vlasov-Maxwell system is

$$
\begin{gathered}
f_{t}+\xi_{2} f_{x_{2}}+\left(E_{1}+\xi_{2} B_{3}\right) f_{\xi_{1}}+\left(E_{2}-\xi_{1} B_{3}\right) f_{\xi_{2}}=0, \\
\frac{\partial B_{3}}{\partial t}=\frac{\partial E_{1}}{\partial x_{2}}, \quad \frac{\partial E_{1}}{\partial t}=\frac{\partial B_{3}}{\partial x_{2}}-j_{1}, \quad \frac{\partial E_{2}}{\partial t}=-j_{2},
\end{gathered}
$$

where

$$
j_{1}=\int_{-\infty}^{\infty} \int_{-\infty}^{\infty} f\left(x_{2}, \xi_{1}, \xi_{2}, t\right) \xi_{1} d \xi_{1} d \xi_{2}, \quad j_{2}=\int_{-\infty}^{\infty} \int_{-\infty}^{\infty} f\left(x_{2}, \xi_{1}, \xi_{2}, t\right) \xi_{2} d \xi_{1} d \xi_{2} .
$$

The initial conditions are given by

$$
\begin{aligned}
f\left(x_{2}, \xi_{1}, \xi_{2}, 0\right) & =\frac{1}{\pi \beta} e^{-\xi_{2}^{2} / \beta}\left[\delta e^{-\left(\xi_{1}-v_{0,1}\right)^{2} / \beta}+(1-\delta) e^{-\left(\xi_{1}+v_{0,2}\right)^{2} / \beta}\right], \\
E_{1}\left(x_{2}, \xi_{1}, \xi_{2}, 0\right) & =E_{2}\left(x_{2}, \xi_{1}, \xi_{2}, 0\right)=0, \quad B_{3}\left(x_{2}, \xi_{1}, \xi_{2}, 0\right)=b \sin \left(k_{0} x_{2}\right),
\end{aligned}
$$


which for $b=0$ is an equilibrium state composed of counter-streaming beams propagating perpendicular to the direction of inhomogeneity. Following [9, we trigger the instability by taking $\beta=0.01, b=0.001$ (the amplitude of the initial perturbation to the magnetic field). Here, $\Omega_{x}=\left[0, L_{y}\right]$, where $L_{y}=2 \pi / k_{0}$, and we set $\Omega_{\xi}=[-1.2,1.2]^{2}$. Two different sets of parameters will be considered,

$$
\begin{aligned}
& \text { choice } 1: \delta=0.5, v_{0,1}=v_{0,2}=0.3, k_{0}=0.2 \\
& \text { choice } 2: \delta=1 / 6, v_{0,1}=0.5, v_{0,2}=0.1, k_{0}=0.2 \text {. }
\end{aligned}
$$

For comparison, these are chosen to correspond to runs of $[9]$.

Accuracy test: The VM system is time reversible, and this provides a way to test the accuracy of our scheme. In particular, let $f(\mathbf{x}, \xi, 0), \mathbf{E}(\mathbf{x}, 0), \mathbf{B}(\mathbf{x}, 0)$ denote the initial conditions for the VM system and $f(\mathbf{x}, \xi, T), \mathbf{E}(\mathbf{x}, T), \mathbf{B}(\mathbf{x}, T)$ the solution at $t=T$. If we choose $f(\mathbf{x},-\xi, T), \mathbf{E}(\mathbf{x}, T),-\mathbf{B}(\mathbf{x}, T)$ as the initial condition at $t=0$, then at $t=T$ we theoretically must recover $f(\mathbf{x},-\xi, 0), \mathbf{E}(\mathbf{x}, 0),-\mathbf{B}(\mathbf{x}, 0)$. In Tables 5.1. 5.2 . we show the $L^{2}$ errors and orders of the numerical solutions with three flux choices for the Maxwell's equations: the upwind flux, the central flux, and one of the alternating fluxes $\widetilde{\mathbf{E}_{h}}=\mathbf{E}_{h}^{+}$and $\widetilde{\mathbf{B}_{h}}=\mathbf{B}_{h}^{-}$. The parameters are those of choice 1 , with symmetric counter-streaming. In the numerical simulations, the third order TVD Runge Kutta time discretization is used, with the CFL number $C_{\mathrm{cfl}}=0.19$ for the upwind and central fluxes, and $C_{\text {cfl }}=0.12$ for the alternating flux in $P^{1}$ and $P^{2}$ cases. For $P^{3}$, we take $\triangle t=O\left(\triangle x^{4 / 3}\right)$ to ensure that the spatial and temporal accuracy is of the same order. From Tables 5.1 . 5.2 . we observe that the schemes with the upwind and alternating fluxes achieve optimal $(k+1)$-th order accuracy in approximating the solution, while for odd $k$, the central flux gives suboptimal approximation of some of the solution components.

TABLE 5.1

Upwind flux for Maxwell's equations, $L^{2}$ errors and orders. Run to $T=5$ and back to $T=10$.

\begin{tabular}{|c|c|c|c|c|c|c|}
\hline \multirow{2}{*}{ Space } & & Mesh=20 & \multicolumn{2}{|c|}{ Mesh=40 } & \multicolumn{2}{c|}{ Mesh=80 } \\
\cline { 3 - 7 } & & error & error & order & error & order \\
\hline \multirow{5}{*}{$\mathcal{G}_{h}^{1}, \mathcal{U}_{h}^{1}$} & $f$ & $0.18 \mathrm{E}+00$ & $0.50 \mathrm{E}-01$ & 1.82 & $0.13 \mathrm{E}-01$ & 1.96 \\
\cline { 2 - 7 } & $B_{3}$ & $0.26 \mathrm{E}-05$ & $0.66 \mathrm{E}-06$ & 2.01 & $0.16 \mathrm{E}-06$ & 2.01 \\
\cline { 2 - 7 } & $E_{1}$ & $0.21 \mathrm{E}-05$ & $0.68 \mathrm{E}-06$ & 1.61 & $0.19 \mathrm{E}-06$ & 1.81 \\
\cline { 2 - 7 } & $E_{2}$ & $0.10 \mathrm{E}-05$ & $0.22 \mathrm{E}-06$ & 2.23 & $0.22 \mathrm{E}-07$ & 3.29 \\
\hline \multirow{5}{*}{$\mathcal{G}_{h}^{2}, \mathcal{U}_{h}^{2}$} & $f$ & $0.56 \mathrm{E}-01$ & $0.77 \mathrm{E}-02$ & 2.87 & $0.10 \mathrm{E}-02$ & 2.92 \\
\cline { 2 - 7 } & $B_{3}$ & $0.23 \mathrm{E}-06$ & $0.26 \mathrm{E}-07$ & 3.12 & $0.32 \mathrm{E}-08$ & 3.06 \\
\cline { 2 - 7 } & $E_{1}$ & $0.16 \mathrm{E}-06$ & $0.16 \mathrm{E}-07$ & 3.32 & $0.14 \mathrm{E}-08$ & 3.54 \\
\cline { 2 - 7 } & $E_{2}$ & $0.16 \mathrm{E}-06$ & $0.22 \mathrm{E}-07$ & 2.90 & $0.15 \mathrm{E}-08$ & 3.91 \\
\hline \multirow{5}{*}{$\mathcal{G}_{h}^{3}, \mathcal{U}_{h}^{3}$} & $f$ & $0.12 \mathrm{E}-01$ & $0.10 \mathrm{E}-02$ & 3.56 & $0.70 \mathrm{E}-04$ & 3.90 \\
\cline { 2 - 7 } & $B_{3}$ & $0.97 \mathrm{E}-07$ & $0.23 \mathrm{E}-08$ & 5.37 & $0.12 \mathrm{E}-09$ & 4.34 \\
\cline { 2 - 7 } & $E_{1}$ & $0.19 \mathrm{E}-07$ & $0.27 \mathrm{E}-09$ & 6.16 & $0.57 \mathrm{E}-11$ & 5.54 \\
\cline { 2 - 7 } & $E_{2}$ & $0.14 \mathrm{E}-07$ & $0.79 \mathrm{E}-09$ & 4.11 & $0.16 \mathrm{E}-10$ & 5.64 \\
\hline
\end{tabular}

Conservation properties: The purpose here is to validate our theoretical result about conservation through two numerical examples, the symmetric case and the non-symmetric case. We first use parameter choice 1 as in the Califano et al. 9], the symmetric case with three different fluxes for Maxwell's equations. The results are illustrated in Figure 5.1. In all the plots, we have rescaled the macroscopic quantities by the physical domain size. For all three fluxes, the mass (charge) is well conserved. The largest relative error for the charge for all three fluxes is smaller than $4 \times 10^{-10}$. As for the total energy, we could observe relatively larger decay in the total energy from the simulation with the upwind flux compared to the one with the other two fluxes. This is expected from the analysis in Section 3. In fact, the largest relative error for the total energy is bounded by $1 \times 10^{-4}$ for th e upwind flux, and bounded by 1 times $10^{-7}$ for central and alternating fluxes.

As for momentum conservation, it is well known that the two species VM system conserves the following expression for the total linear momentum:

$$
\mathbf{P}=\int \xi f d \xi d \mathbf{x}+\int \mathbf{E} \times \mathbf{B} d \mathbf{x}
$$


TABLE 5.2

Central and alternating fluxes for Maxwell's equations, $L^{2}$ errors and orders. Run to $T=5$ and back to $T=10$.

\begin{tabular}{|c|c|c|c|c|c|c|c|c|c|c|c|}
\hline & & \multicolumn{5}{|c|}{ Central } & \multicolumn{5}{|c|}{ Alternating } \\
\hline & & \multirow{2}{*}{$\begin{array}{c}\text { Mesh }=20^{3} \\
\text { error }\end{array}$} & \multicolumn{2}{|c|}{ Mesh $=40^{3}$} & \multicolumn{2}{|c|}{ Mesh $=80^{3}$} & \multirow{2}{*}{$\begin{array}{c}\text { Mesh }=20^{3} \\
\text { error }\end{array}$} & \multicolumn{2}{|c|}{ Mesh $=40^{3}$} & \multicolumn{2}{|c|}{ Mesh $=80^{3}$} \\
\hline & & & error & order & error & order & & error & order & error & order \\
\hline \multirow{2}{*}{$\mathcal{G}_{h}^{1}$} & $f$ & $0.18 \mathrm{E}+00$ & $0.50 \mathrm{E}-01$ & 1.82 & $0.13 \mathrm{E}-01$ & 1.96 & $0.18 \mathrm{E}+00$ & $0.50 \mathrm{E}-01$ & 1.82 & $0.13 \mathrm{E}-01$ & 1.96 \\
\hline & $B_{3}$ & $0.13 \mathrm{E}-04$ & $0.85 \mathrm{E}-05$ & 0.66 & $0.50 \mathrm{E}-05$ & 0.75 & $0.29 \mathrm{E}-05$ & $0.78 \mathrm{E}-06$ & 1.90 & $0.22 \mathrm{E}-06$ & 1.83 \\
\hline \multirow{2}{*}{$\mathcal{U}_{h}^{1}$} & $E_{1}$ & $0.19 \mathrm{E}-05$ & $0.13 \mathrm{E}-05$ & 0.51 & $0.58 \mathrm{E}-06$ & 1.17 & $0.24 \mathrm{E}-06$ & $0.35 \mathrm{E}-07$ & 2.74 & $0.22 \mathrm{E}-08$ & 3.99 \\
\hline & $E_{2}$ & $0.92 \mathrm{E}-06$ & $0.19 \mathrm{E}-06$ & 2.26 & $0.20 \mathrm{E}-07$ & 3.24 & $0.10 \mathrm{E}-05$ & $0.22 \mathrm{E}-06$ & 2.23 & $0.22 \mathrm{E}-07$ & 3.29 \\
\hline \multirow{2}{*}{$\mathcal{G}_{h}^{2}$} & $f$ & $0.56 \mathrm{E}-01$ & $0.77 \mathrm{E}-02$ & 2.87 & $0.10 \mathrm{E}-02$ & 2.92 & $0.56 \mathrm{E}-01$ & $0.77 \mathrm{E}-02$ & 2.87 & $0.10 \mathrm{E}-02$ & 2.92 \\
\hline & $B_{3}$ & $0.28 \mathrm{E}-06$ & $0.28 \mathrm{E}-07$ & 3.34 & $0.32 \mathrm{E}-08$ & 3.15 & $0.28 \mathrm{E}-06$ & $0.22 \mathrm{E}-07$ & 3.70 & $0.18 \mathrm{E}-08$ & 3.63 \\
\hline \multirow{2}{*}{$\mathcal{U}_{h}^{2}$} & $E_{1}$ & $0.18 \mathrm{E}-07$ & $0.56 \mathrm{E}-09$ & 5.00 & $0.88 \mathrm{E}-11$ & 5.99 & $0.32 \mathrm{E}-07$ & $0.30 \mathrm{E}-09$ & 6.72 & $0.11 \mathrm{E}-10$ & 4.84 \\
\hline & $E_{2}$ & $0.16 \mathrm{E}-06$ & $0.22 \mathrm{E}-07$ & 2.90 & $0.15 \mathrm{E}-08$ & 3.91 & $0.16 \mathrm{E}-06$ & $0.22 \mathrm{E}-07$ & 2.90 & $0.15 \mathrm{E}-08$ & 3.91 \\
\hline \multirow{2}{*}{$\mathcal{G}_{h}^{3}$} & $f$ & $0.12 \mathrm{E}-01$ & $0.10 \mathrm{E}-02$ & 3.56 & $0.70 \mathrm{E}-04$ & 3.90 & $0.12 \mathrm{E}-01$ & $0.10 \mathrm{E}-02$ & 3.56 & $0.70 \mathrm{E}-04$ & 3.90 \\
\hline & $B_{3}$ & $0.10 \mathrm{E}-06$ & $0.44 \mathrm{E}-08$ & 4.57 & $0.16 \mathrm{E}-09$ & 4.81 & $0.10 \mathrm{E}-06$ & $0.24 \mathrm{E}-08$ & 5.42 & $0.12 \mathrm{E}-09$ & 4.36 \\
\hline \multirow{2}{*}{$\mathcal{U}_{h}^{3}$} & $E_{1}$ & $0.46 \mathrm{E}-07$ & $0.82 \mathrm{E}-10$ & 9.12 & $0.30 \mathrm{E}-10$ & 1.45 & $0.98 \mathrm{E}-08$ & $0.10 \mathrm{E}-09$ & 6.60 & $0.90 \mathrm{E}-12$ & 6.80 \\
\hline & $E_{2}$ & $0.14 \mathrm{E}-07$ & 0.79E-09 & 4.12 & $0.16 \mathrm{E}-10$ & 5.65 & $0.14 \mathrm{E}-07$ & $0.79 \mathrm{E}-09$ & 4.11 & $0.16 \mathrm{E}-10$ & 5.64 \\
\hline
\end{tabular}

where the first term represents the momentum in the particles while the second that of the electromagnetic field. In fact, this is true for the full energy-momentum and angular momentum tensors [49. Each component of the spatial integrand of (5.6), the components of the momentum density, satisfies a conservation law, a result that relies on both species being dynamic and one that relies on the constraint equations $(1.1 \mathrm{c})$ being satisfied. However, in this paper we have fixed the constant ion background by charge neutrality and, consequently, momentum is not conserved in general. This lack of conservation does not appear to be widely known, but it is known that the enforcement of constraints may or may not results in the loss of conservation 45. For example, the single species Vlasov-Poisson system with a fixed constant ion background does indeed conserve momentum. However, for the streaming Weibel application, it is not diffi cult to show that the following component is conserved:

$$
P_{1}=\int \xi_{1} f d \xi_{1} d \xi_{2} d x_{2}+\int E_{2} B_{3} d x_{2}
$$

while the component $P_{2}$ is not. Since conservation of $P_{1}$ relies on the constraint equations and since our computational algorithm does not enforce these constraints, conservation of $P_{1}$ serves as a measure of the goodness of our method in maintaining the initial satisfaction of the constraints. From Figure 5.1, we see that all three flux formulations conserve $P_{1}$ relatively well, but, as expected, there is a large accumulating error in $P_{2}$, particularly for the alternating flux case.

Similarly, for a general VM system without constraints, the following expression for the total angular momentum is conserved:

$$
\mathbf{L}=\int \mathbf{x} \times \xi f d \xi d \mathbf{x}+\int \mathbf{x} \times(\mathbf{E} \times \mathbf{B}) d \mathbf{x}
$$

However, because the SW application breaks symmetry, there is no relevant component of the angular momentum that is conserved for this problem, but for a more general application one may want to track its conservation.

Comparison and interpretation: In Figure 5.3. we plot the time evolution of the kinetic, electric, and magnetic energies. In particular, we plot the separate components defined by $K_{1}=\frac{1}{2} \int f \xi_{1}^{2} d \xi_{1} d \xi_{2} d x_{2}$, $K_{2}=\frac{1}{2} \int f \xi_{2}^{2} d \xi_{1} d \xi_{2} d x_{2}, \mathrm{E}_{1}=\frac{1}{2} \int E_{1}^{2} d x_{2}$, and $\mathrm{E}_{2}=\frac{1}{2} \int E_{2}^{2} d x_{2}$. Figure (a) shows for choice 1 the transference of kinetic energy from one component to the other with a deficit converted into field energy. This deficit is consistent with energy conservation, as evidenced by Figure 5.1. Observe the magnetic and inductive electric fields grow initially at a linear growth rate (comparable to that of Table I of [9]). Saturation occurs when the electric and magnetic energies simultaneously peak at around $t=70$ in agreement with [9; however, in our case we achieve equipartition at the peak, which may be due to better resolution. Here we have also shown the longitudinal component $E_{2}$, not shown in [9, which in Figure (b) is seen to grow at twice the 
growth rate. This behavior was anticipated in [10] in the context of a two-fluid model and seen in kinetic VM computations of the usual Weibel instability 47. It is due to wave coupling and a modulation of the electron density induced by the spatial modulation of $B_{3}^{2}$. The growth at twice the growth rate of the magnetic field $B_{3}$ is seen in Figure (b), and the density modulation, including the expected spikes, is seen in Figure 5.4 We have also calculated the first four Log Fourier modes of the fields $E_{1}, E_{2}, B_{3}$, and these are shown in Figure 5.5. Here, the $n$-th Log Fourier mode for a function $W(x, t)[34$ is defined as

$$
\log F M_{n}(t)=\log _{10}\left(\frac{1}{L} \sqrt{\left|\int_{0}^{L} W(x, t) \sin (k n x) d x\right|^{2}+\left|\int_{0}^{L} W(x, t) \cos (k n x) d x\right|^{2}}\right) .
$$

In Figures 5.6 we plot the 2D contours of $f$ at selected locations $x_{2}$ and time $t$, when the upwind flux is used in the Maxwell solver. The times chosen correspond to those for the density of Figure 5.4 and we see that at late times considerable fine structure is present, which is consistent with the Log Fourier plots. For completeness, we also include in Figure 5.2 plots of the electric and magnetic fields at the final time for our three fluxes.

For choice 2, with the nonsymmetric parameter set, the results are included in Figures 5.2, 5.3, 5.4, 5.5 , and 5.6. juxtaposed with those for parameter choice 1. Insofar as we can make comparison with [9], our results are in reasonable agreement. Similar energy transfers take place, but the equipartition of the magnetic and electric energies at the peak is not achieved. All modes saturate now at nearly the same values, evidently resulting from the broken symmetry. Also, at long times, contours of the distribution function are displayed. Here the wrapping of the distribution function as two intertwined distorted cylinders is observed as in 9], although for late times there is a loss of localization.

6. Concluding Remarks. In summary, we have developed discontinuous Galerkin methods for solving the Vlasov-Maxwell system. We have proven that the method is arbitrarily accurate, conserves charge, can conserve energy, and is stable. Error estimates were established for several flux choices. The scheme was tested on the streaming Weibel instability, where the order of accuracy and conservation properties were verified. In the future, we will explore other time stepping methods to improve the efficiency of the overall algorithm. In our development, the constraint equations of $1.1 \mathrm{c}$ were not considered; in the future, we plan to investigate them together with some correction techniques for the continuity equation. The proposed method has been clearly established as sufficient for investigating the streaming Weibel instability, and the long time nonlinear physics of this system can be further investigated and modeled. In the future, we will also apply the method to study other important plasma physics problems, especially those of higher dimension.

Acknowledgments. Y.C. is supported by grant NSF DMS-1217563, I.M.G. is supported by grant NSF DMS-1109625, and F.L. is partially supported by NSF CAREER award DMS-0847241 and an Alfred P. Sloan Research Fellowship. P.J.M is supported by the US Department of Energy, grant DE-FG02-04ER54742; He would like to thank F. Pegoraro for helpful correspondence. Also, support from Department of Mathematics at Michigan State University and the Institute of Computational Engineering and Sciences at the University of Texas Austin are gratefully acknowledged.

\section{REFERENCES}

[1] M. Ainsworth. Dispersive and dissipative behavior of high order discontinuous Galerkin finite element methods. J. Comp. Phys., 198:106-130, 2004.

[2] B. Ayuso, J. A. Carrillo, and C.-W. Shu. Discontinuous Galerkin methods for the multi-dimensional Vlasov-Poisson problems. Mathematical Models and Methods in Applied Sciences. to appear.

[3] B. Ayuso, J. A. Carrillo, and C.-W. Shu. Discontinuous Galerkin methods for the one-dimensional Vlasov-Poisson system. Kinetic and Related Models, 4:955-989, 2011.

[4] N. Besse, G. Latu, A. Ghizzo, E. Sonnendrüker, and P. Bertrand. A wavelet-MRA-based adaptive semi-Lagrangian method for the relativistic Vlasov-Maxwell system. J. Comp. Phys., 227(16):7889 - 7916, 2008.

[5] C. K. Birdsall and A. B. Langdon. Plasma physics via computer simulation. Institute of Physics Publishing, 1991.

[6] J. Boris and D. Book. Solution of continuity equations by the method of flux-corrected transport. J. Comp. Phys., 20:397-431, 1976.

[7] F. Califano, N. Attico, F. Pegoraro, G. Bertin, and S. V. Bulanov. Fast formation of magnetic islands in a plasma in the presence of counterstreaming electrons. Phys. Rev. Lett., 86(23):5293-5296, 2001. 


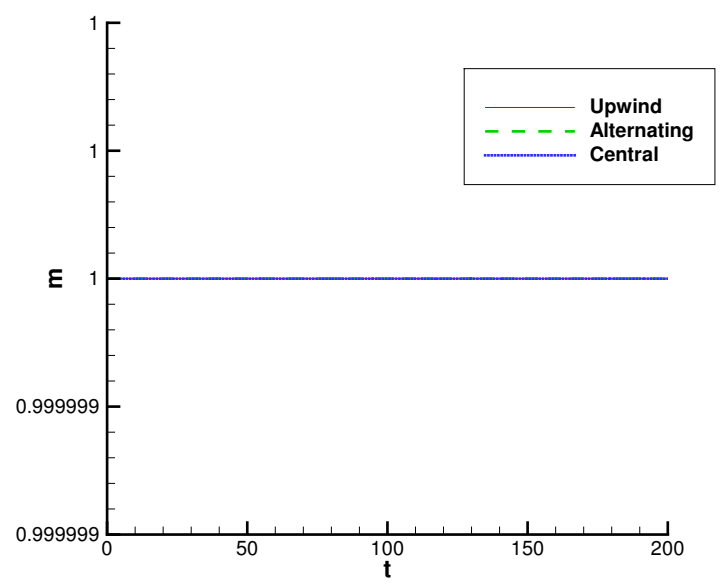

(a) Mass

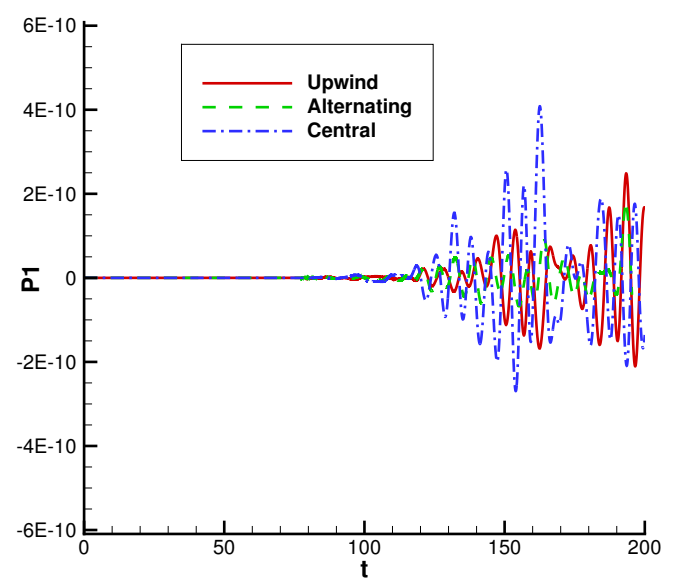

(c) Momentum $P_{1}$

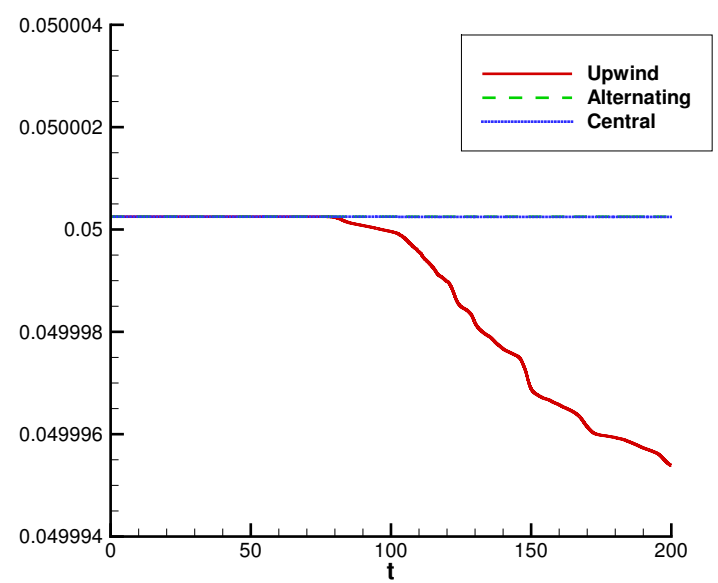

(b) Total energy

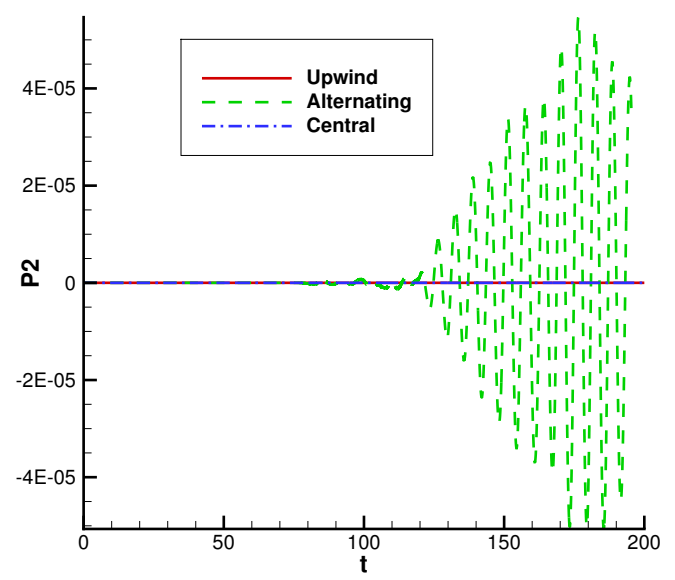

(d) Momentum $P_{2}$

FIG. 5.1. Streaming Weibel instability with parameter choice 1 as in Califano et al. [9] $\left(\delta=0.5, v_{0,1}=v_{0,2}=0.3, k_{0}=\right.$ $0.2)$, the symmetric case. The mesh is $100^{3}$ with piecewise quadratic polynomials. Time evolution of mass, total energy, and momentum for the three numerical fluxes for the Maxwell's equations.

[8] F. Califano, F. Pegoraro, and S. V. Bulanov. Impact of kinetic processes on the macroscopic nonlinear evolution of the electromagnetic-beam-plasma instability. Phys. Rev. Lett., 84:3602-3605, 2000.

[9] F. Califano, F. Pegoraro, S. V. Bulanov, and A. Mangeney. Kinetic saturation of the Weibel instability in a collisionless plasma. Phys. Rev. E, 57(6):7048-7059, 1998.

[10] F. Califano, R. Prandi, F. Pegoraro, and S. V. Bulanov. Magnetic-field generation and wave-breaking in collisionless plasmas. J. Plasma Phys., 60:331-339, 1998.

[11] C. Z. Cheng and G. Knorr. The integration of the Vlasov equation in configuration space. J. Comp. Phys., 22(3):330-351, 1976.

[12] Y. Cheng and I. M. Gamba. Numerical study of Vlasov-Poisson equations for infinite homogeneous stellar systems. Comm. Nonlin. Sci. Num. Sim., 17, 2012.

[13] Y. Cheng, I. M. Gamba, and P. J. Morrison. Study of conservation and recurrence of Runge-Kutta discontinuous Galerkin schemes for Vlasov-Poisson systems. J. Sci. Comp. accepted, 2012. preprint arXiv:1209.6413v2 [math.NA].

[14] P. Ciarlet. The finite element methods for elliptic problems. North-Holland, Amsterdamk, 1975.

[15] B. Cockburn, S. Hou, and C.-W. Shu. The Runge-Kutta local projection discontinuous Galerkin finite element method for conservation laws IV: the multidimensional case. Math. Comput., 54:545-581, 1990.

[16] B. Cockburn, S. Y. Lin, and C.-W. Shu. TVB Runge-Kutta local projection discontinuous Galerkin finite element method 


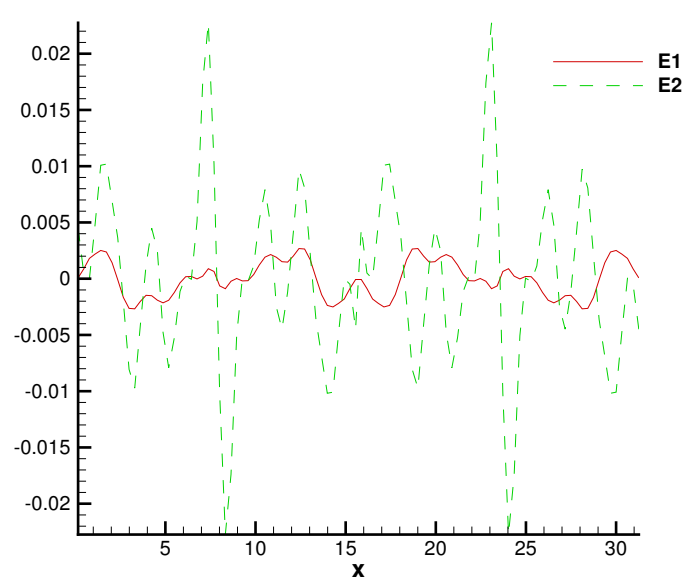

(a) Parameter Choice 1. Electric field, upwind flux

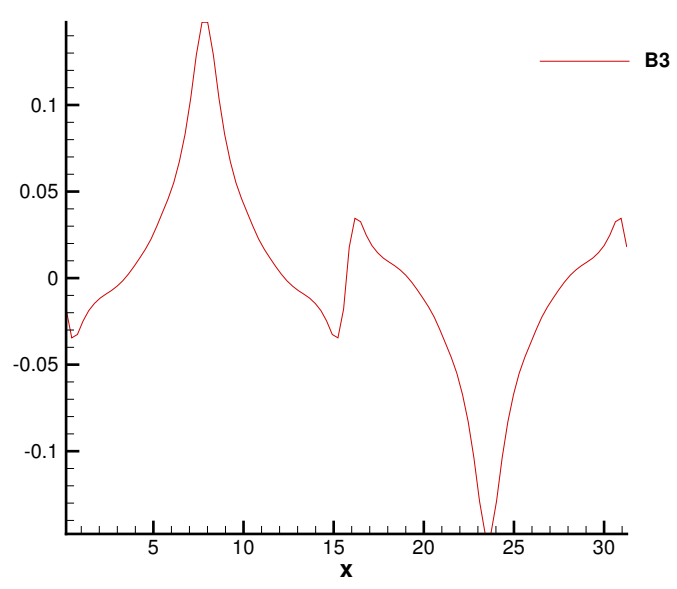

(c) Parameter Choice 1. Magnetic field, upwind flux

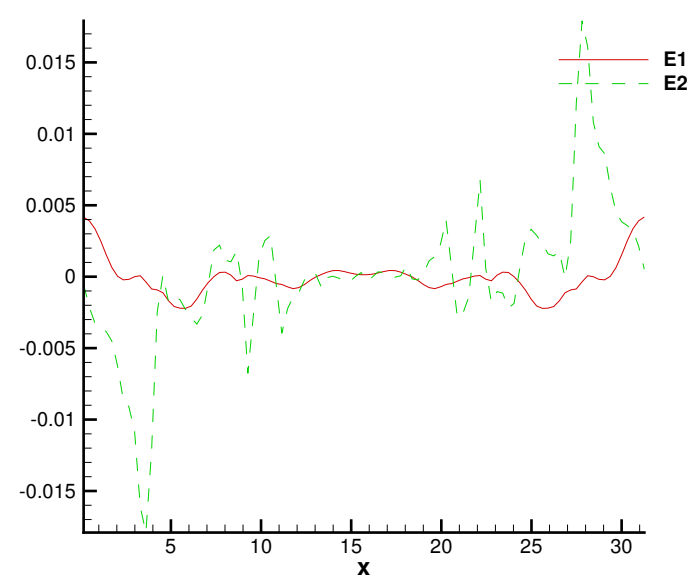

(b) Parameter Choice 2. Electric field, upwind flux

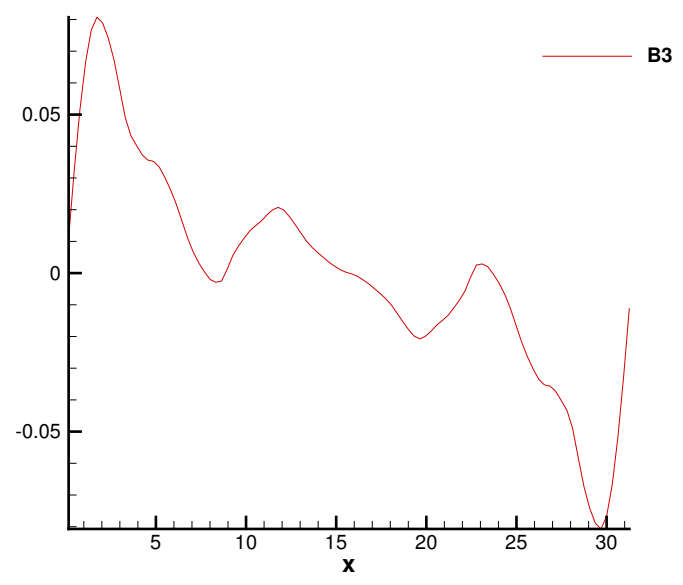

(d) Parameter Choice 2. Magnetic field, upwind flux

FIG. 5.2. Streaming Weibel instability. The mesh is $100^{3}$ with piecewise quadratic polynomials. The electric and magnetic fields at $T=200$.

for conservation laws III: one dimensional systems. J. Comput. Phys., 84:90-113, 1989.

[17] B. Cockburn and C.-W. Shu. TVB Runge-Kutta local projection discontinuous Galerkin finite element method for conservation laws II: general framework. Math. Comput., 52:411-435, 1989.

[18] B. Cockburn and C.-W. Shu. The Runge-Kutta local projection p1-discontinuous Galerkin finite element method for scalar conservation laws. Math. Model. Num. Anal., 25:337-361, 1991.

[19] B. Cockburn and C.-W. Shu. The Runge-Kutta discontinuous Galerkin method for conservation laws V: multidimensional systems. J. Comput. Phys., 141:199-224, 1998.

[20] M. Crouzeix and V. Thomée. The stability in $L_{p}$ and $W_{p}^{1}$ of the $L_{2}$-projection onto finite element function spaces. Mathematics of Computation, 178:521-532, 1987.

[21] R. DiPerna and P.-L. Lions. Global weak solutions of Vlasov-Maxwell systems. Communication on Pure and Applied Mathematics, 42:729-757, 1989.

[22] E. G. Evstatiev and B. A. Shadwick. Variational formulation of particle algorithms for kinetic plasma simulations. 2012. preprint arXiv:1210.3743 [physics.plasm-ph].

[23] E. Fijalkow. A numerical solution to the Vlasov equation. Comput. Phys. Comm., 116:319-328, 1999.

[24] F. Filbet, E. Sonnendrücker, and P. Bertrand. Conservative numerical schemes for the Vlasov equation. J. Comp. Phys., 172:166-187, 2001.

[25] R. Glassey and J. Schaeffer. Global existence for the relativistic Vlasov-Maxwell system with nearly neutral initial data. 


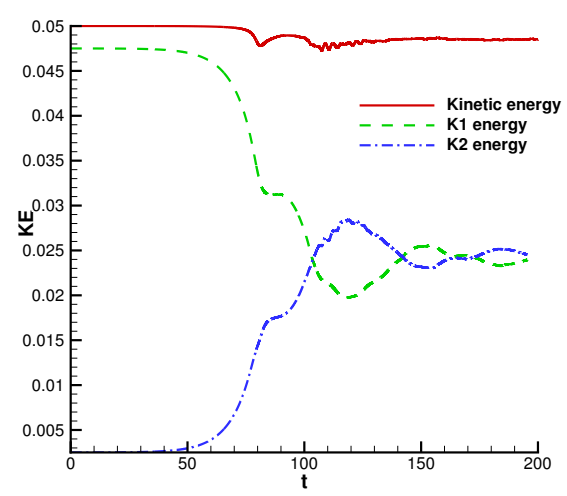

(a) Parameter Choice 1. Kinetic energies

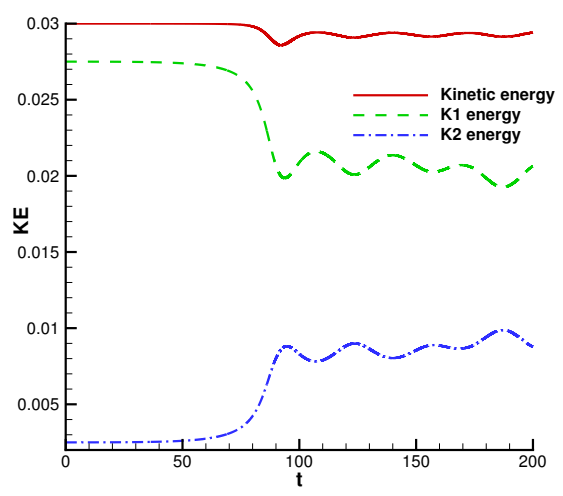

(c) Parameter Choice 2. Kinetic energies

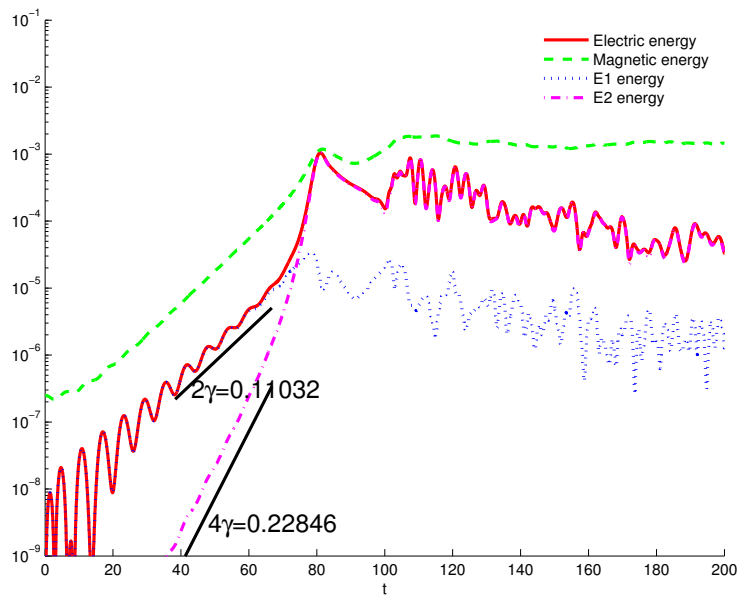

(b) Parameter Choice 1. Field energies

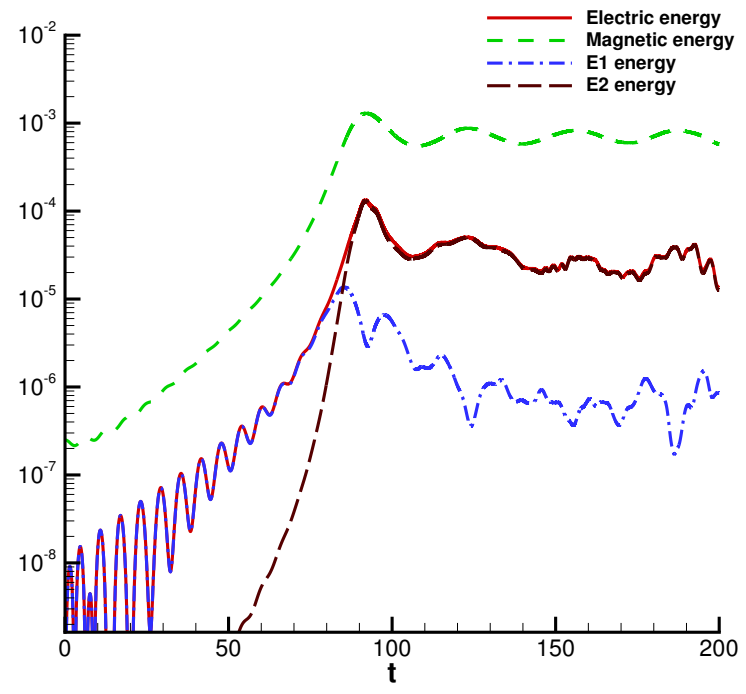

(d) Parameter Choice 2. Field energies

FIG. 5.3. Streaming Weibel instability. The mesh is $100^{3}$ with piecewise quadratic polynomials. Time evolution of kinetic, electric and magnetic energies by alternating flux for the Maxwell's equations.

Communications in Mathematical Physics, 119:353-384, 1988.

[26] R. Glassey and J. Schaeffer. The "two and one-half-dimensional" relativistic Vlasov Maxwell system. Communications in Mathematical Physics, 185:257-284, 1997.

[27] R. Glassey and J. Schaeffer. The relativistic Vlasov-Maxwell system in two space dimensions. I. Archive for Rational Mechanics and Analysis, 141:331-354, 1998.

[28] R. Glassey and J. Schaeffer. The relativistic Vlasov-Maxwell system in two space dimensions. II. Archive for Rational Mechanics and Analysis, 141:355-374, 1998.

[29] R. T. Glassey and W. A. Strauss. Singularity formation in a collisionless plasma could occur only at high velocities. Archive for Rational Mechanics and Analysis, 92:59-90, 1986.

[30] R. T. Glassey and W. A. Strauss. Absence of shocks in an initially dilute collisionless plasma. Communications in Mathematical Physics, 113:191-208, 1987.

[31] S. Gottlieb and C.-W. Shu. Total variation diminishing Runge-Kutta schemes. Math. Comput., 67:73-85, 1998.

[32] S. Gottlieb, C.-W. Shu, and E. Tadmor. Strong stability preserving high order time discretization methods. SIAM Review, 43:89-112, 2001. 
[33] R. E. Heath. Numerical analysis of the discontinuous Galerkin method applied to plasma physics. 2007. Ph. D. dissertation, the University of Texas at Austin.

[34] R. E. Heath, I. M. Gamba, P. J. Morrison, and C. Michler. A discontinuous Galerkin method for the Vlasov-Poisson system. J. Comp. Phys., 231:1140-1174, 2012.

[35] R. W. Hockney and J. W. Eastwood. Computer simulation using particles. McGraw-Hill, New York, 1981.

[36] F. Huot, A. Ghizzo, P. Bertrand, E. Sonnendrüker, and O. Coulaud. Instability of the time splitting scheme for the one-dimensional and relativistic Vlasov-Maxwell system. J. Comp. Phys., 185(2):512 - 531, 2003.

[37] G. B. Jacobs and J. S. Hesthaven. High-order nodal discontinuous galerkin particle-in-cell method on unstructured grids. J. Comput. Phys., 214:96-121, May 2006.

[38] G. B. Jacobs and J. S. Hesthaven. Implicit explicit time integration of a high-order particle-in-cell method with hyperbolic divergence cleaning. Computer Physics Communications, 180(10):1760-1767, 2009.

[39] A. J. Klimas. A method for overcoming the velocity space filamentation problem in collisionless plasma model solutions. J. Comp. Phys., 68:202-226, 1987.

[40] A. J. Klimas and W. M. Farrell. A splitting algorithm for Vlasov simulation with filamentation filtration. J. Comp. Phys., 110:150-163, 1994.

[41] P. Lesaint and P.-A. Raviart. On a finite element method for solving the neutron transport equation. In Mathematical aspects of finite elements in partial differential equations (Proc. Sympos., Math. Res. Center, Univ. Wisconsin, Madison, Wis., 1974), pages 89-123. Math. Res. Center, Univ. of Wisconsin-Madison, Academic Press, New York, 1974.

[42] A. Mangeney, F. Califano, C. Cavazzoni, and P. Travnicek. A numerical scheme for the integration of the Vlasov-Maxwell system of equations. J. Comp. Phys., 179(2):495-538, 2002.

[43] P. J. Morrison. The Maxwell-Vlasov equations as a continuous Hamiltonian system. Phys. Lett. A, 80:383-386, 1980.

[44] P. J. Morrison. A general theory for gauge-free lifting. Phys. Plasmas, 80:012104, 2013.

[45] P. J. Morrison, N. Lebovitz, and J. A. Biello. The Hamiltonian description of incompressible fluid ellipsoids. Ann. Phys., 324:1747-1762, 2009.

[46] C.-D. Munz, P. Omnes, R. Schneider, E. Sonnendrücker, and U. Voß. Divergence correction techniques for Maxwell solvers based on a hyperbolic model. J. Comp. Phys., 161:484-511, 2000.

[47] L. Palodhi, F. Califano, and F. Pegoraro. Nonlinear kinetic development of the Weibel instability and the generation of electrostatic coherent structures. Plasma Phys. Control. Fusion, 51:125006, 2009.

[48] F. Pegoraro, S. V. Bulanov, F. Califano, and M. Lontano. Nonlinear development of the Weibel instability and magnetic field generation in collisionless plasmas. Phys. Scripta, T63:262-265, 1996.

[49] D. Pfirsch and P. J. Morrison. Local conservation laws for the Vlasov-Maxwell and collisionless kinetic guiding-center theories. Phys. Rev. A, 32:1714-1721, 1985.

[50] J.-M. Qiu and C.-W. Shu. Positivity preserving semi-Lagrangian discontinuous Galerkin formulation: theoretical analysis and application to the Vlasov-Poisson system. 2011. submitted to J. Comp. Phys.

[51] W. Reed and T. Hill. Triangular mesh methods for the neutron transport equation. Technical report, Los Alamos National Laboratory, Los Alamos, NM, 1973.

[52] J. Rossmanith and D. Seal. A positivity-preserving high-order semi-Lagrangian discontinuous Galerkin scheme for the Vlasov-Poisson equations. 2011. submitted to J. Comp. Phys.

[53] C.-W. Shu and S. Osher. Efficient implementation of essentially non-oscillatory shock-capturing schemes. J. Comput. Phys., 77:439-471, 1988.

[54] N. Sircombe and T. Arber. VALIS: A split-conservative scheme for the relativistic 2d Vlasov-Maxwell system. J. Comp. Phys., 228(13):4773 - 4788, 2009.

[55] E. Sonnendrücker, J. Roche, P. Bertrand, and A. Ghizzo. The semi-Lagrangian method for the numerical resolution of the Vlasov equation. J. Comp. Phys., 149(2):201-220, 1999.

[56] A. Suzuki and T. Shigeyama. A conservative scheme for the relativistic Vlasov-Maxwell system. J. Comp. Phys., 229(5):1643 - 1660, 2010.

[57] E. S. Weibel. Spontaneously growing transverse waves in a plasma due to an anisotropic velocity distribution. Phys. Rev. Lett., 2:83-84, Feb 1959.

[58] S. Zaki, L. Gardner, and T. Boyd. A finite element code for the simulation of one-dimensional Vlasov plasmas. i. theory. J. Comp. Phys., 79:184-199, 1988.

[59] S. Zaki, L. Gardner, and T. Boyd. A finite element code for the simulation of one-dimensional Vlasov plasmas. ii. applications. J. Comp. Phys., 79:200-208, 1988. 


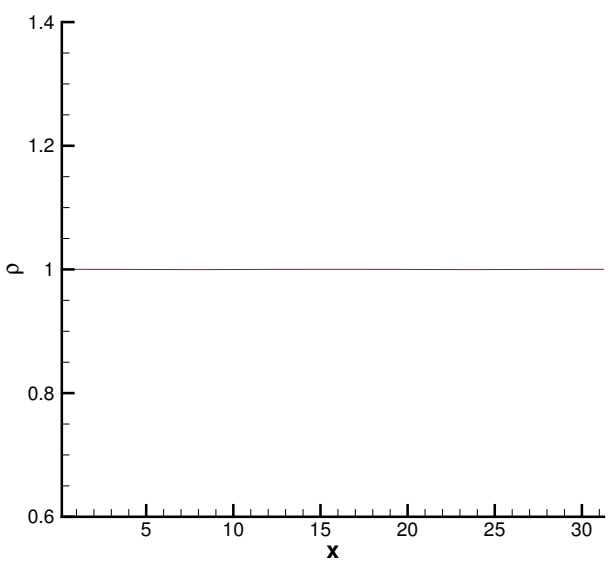

(a) Parameter Choice 1. $t=55$.

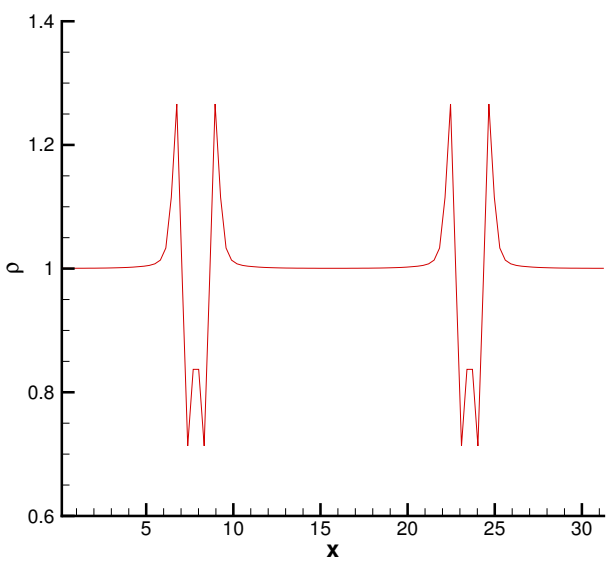

(c) Parameter Choice 1. $t=82$.

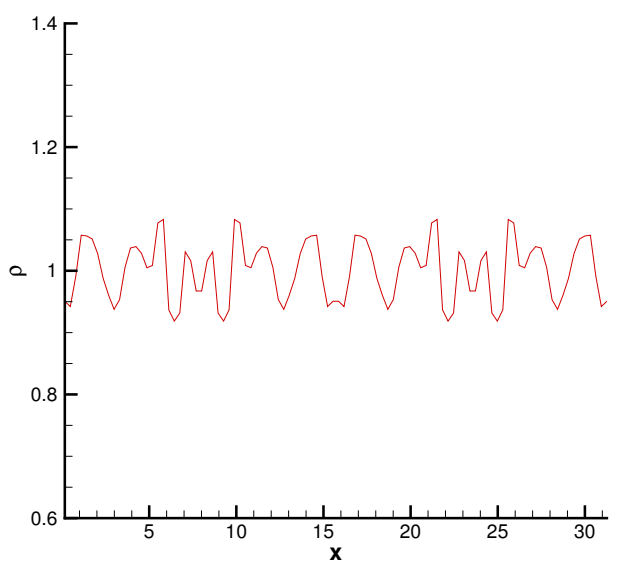

(e) Parameter Choice 1. $t=125$.

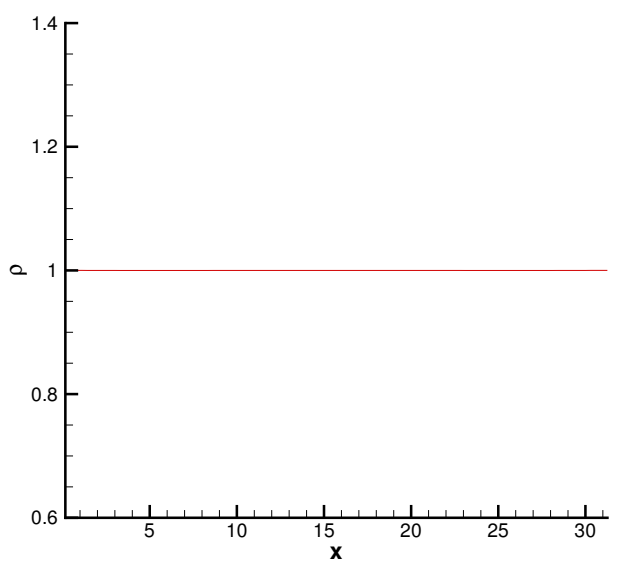

(b) Parameter Choice 2. $t=55$.

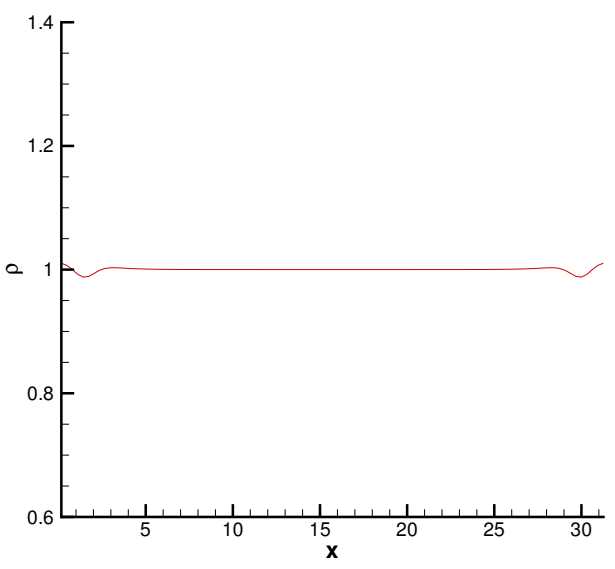

(d) Parameter Choice 2. $t=82$.

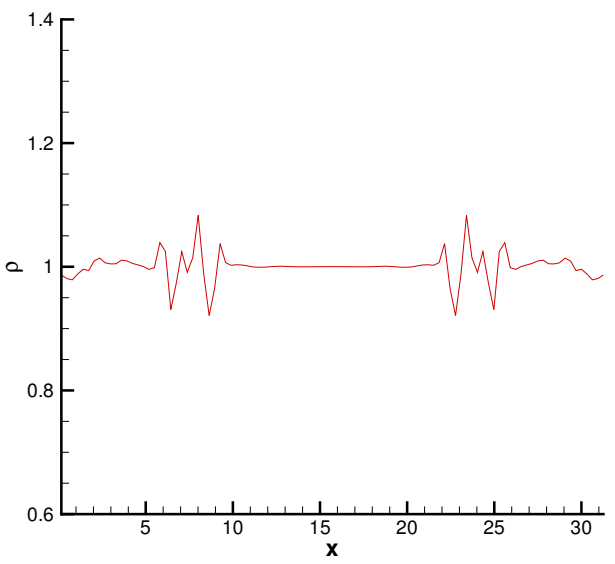

(f) Parameter Choice 2. $t=125$.

FIG. 5.4. Plots of the computed density function $\rho_{h}$ for the streaming Weibel instability at selected time $t$. The mesh is $100^{3}$ with piecewise quadratic polynomials. The upwind flux is applied. 


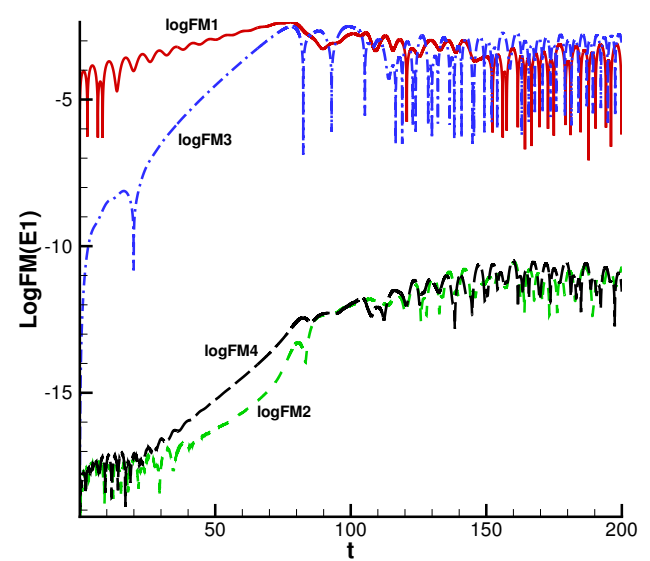

(a) Parameter Choice 1. Log Fourier modes of $E_{1}$

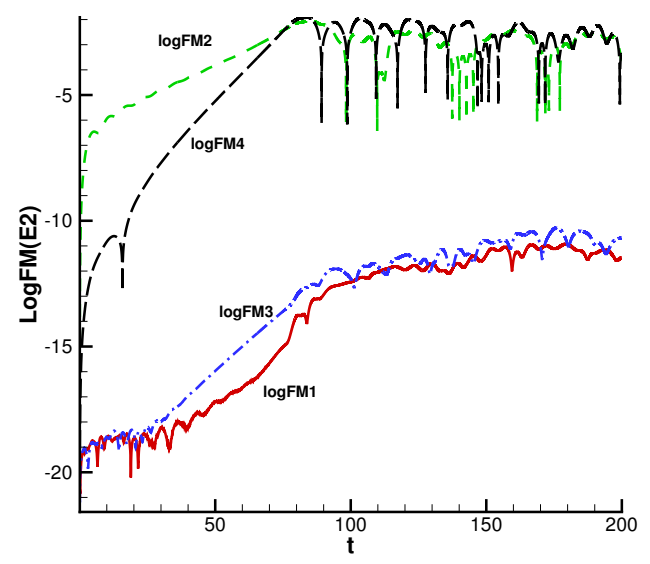

(c) Parameter Choice 1. Log Fourier modes of $E_{2}$

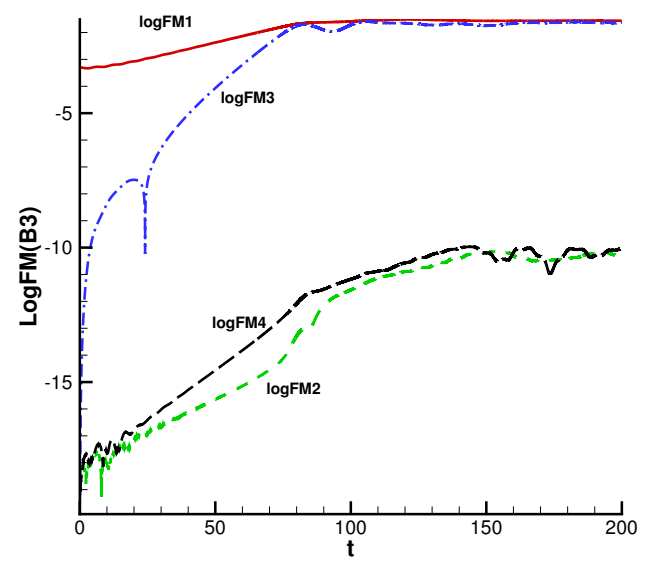

(e) Parameter Choice 1. Log Fourier modes of $B_{3}$

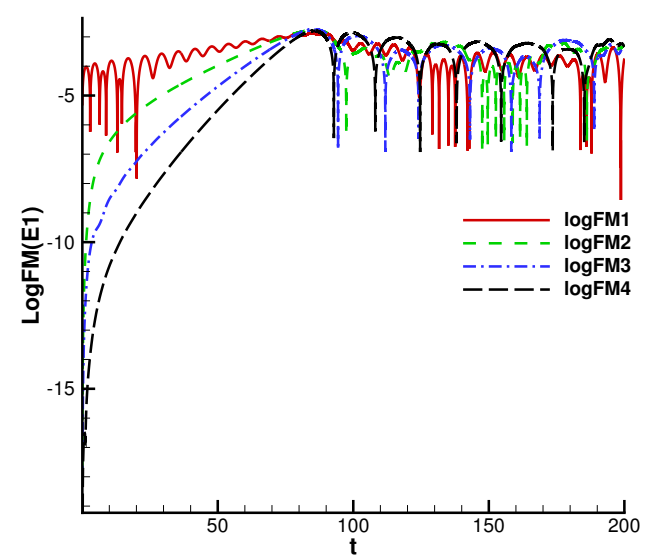

(b) Parameter Choice 2. Log Fourier modes of $E_{1}$

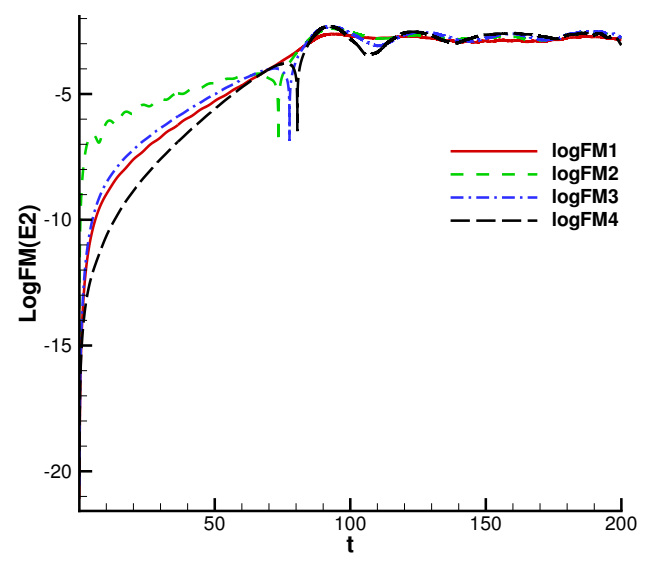

(d) Parameter Choice 2. Log Fourier modes of $E_{2}$

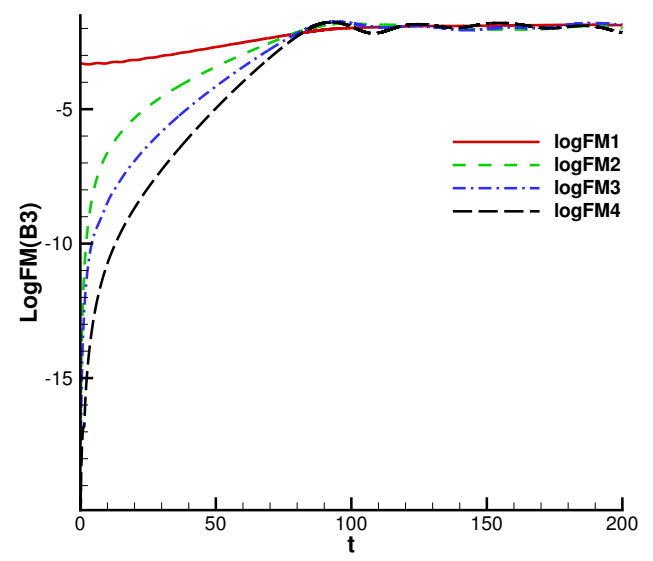

(f) Parameter Choice 2. Log Fourier modes of $B_{3}$

FIG. 5.5. Streaming Weibel instability. The mesh is $100^{3}$ with piecewise quadratic polynomials. The first four Log Fourier modes of $E_{1}, E_{2}, B_{3}$ computed by the alternating flux for the Maxwell's equations. 


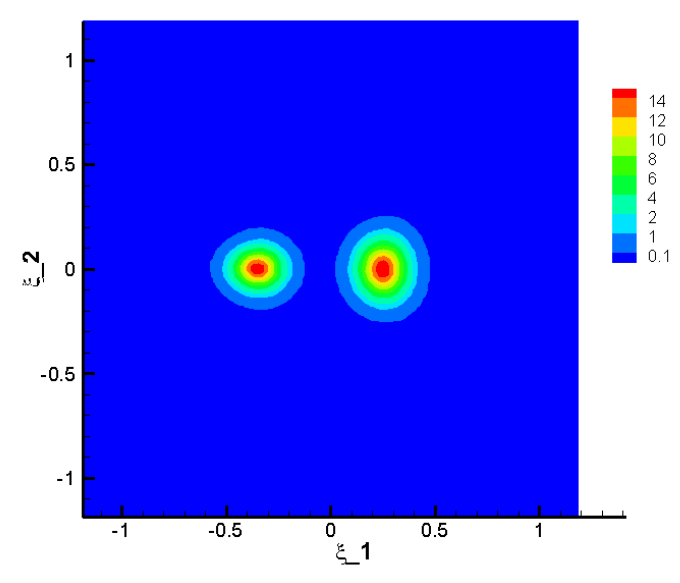

(a) Parameter Choice 1. $x_{2}=0.05 \pi, t=55$.

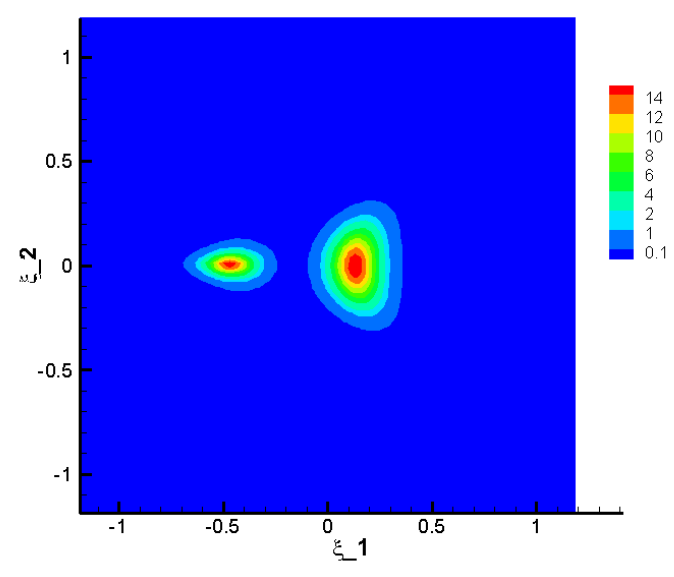

(c) Parameter Choice 1. $x_{2}=0.05 \pi, t=82$.

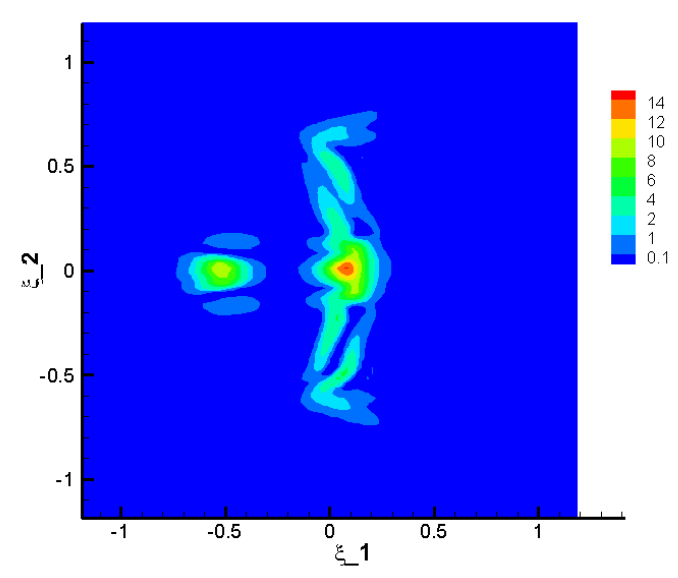

(e) Parameter Choice 1. $x_{2}=0.05 \pi, t=125$.

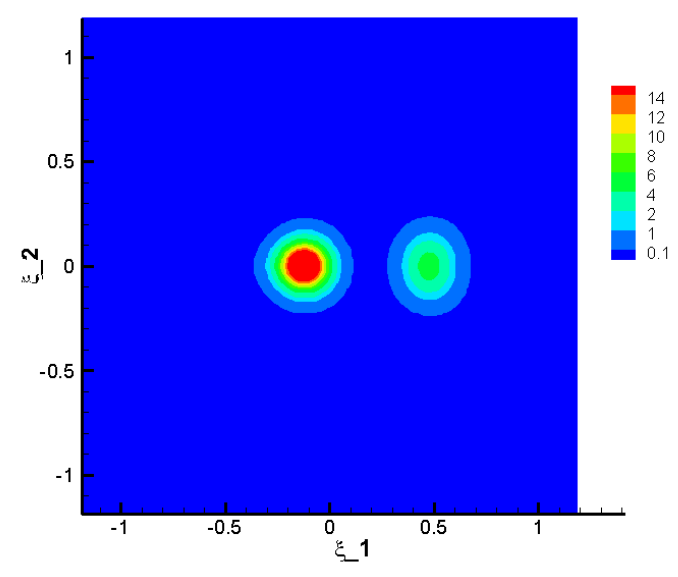

(b) Parameter Choice 2. $x_{2}=0.05 \pi, t=55$.

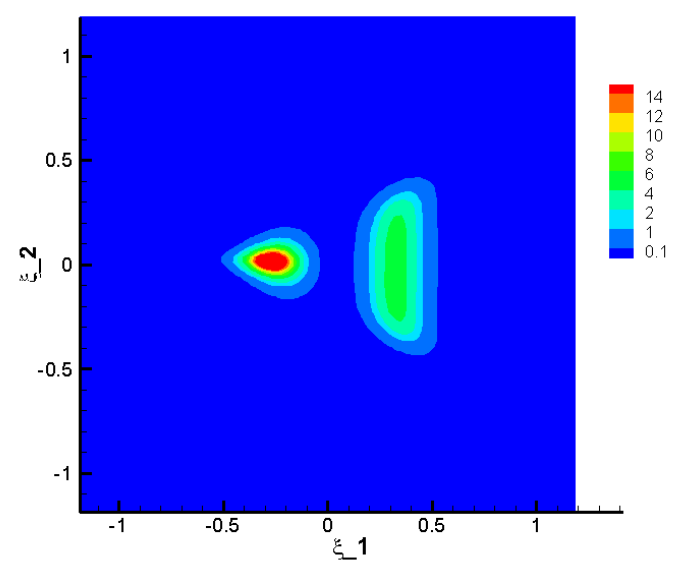

(d) Parameter Choice 2. $x_{2}=0.05 \pi, t=82$.

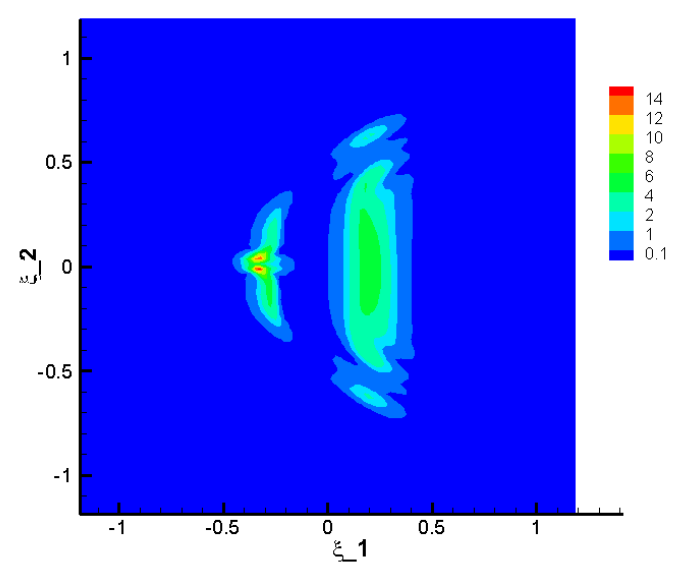

(f) Parameter Choice 2. $x_{2}=0.05 \pi, t=125$.

FIG. 5.6. $2 D$ contour plots of the computed distribution function $f_{h}$ for the streaming Weibel instability. The mesh is $100^{3}$ with piecewise quadratic polynomials. The upwind flux is applied. 